\title{
CLASSIFICATION OF OPERATORS BY MEANS OF THEIR OPERATIONAL CALCULUS
}

\author{
BY \\ SHMUEL KANTOROVITZ $\left({ }^{1}\right)$
}

Introduction. It was realized recently that a natural starting point for spectral theory is the operational calculus (see [7] and [15]). Instead of postulating the existence of a resolution of the identity (as in [4]) one postulates the existence of an operational calculus, which is an extension of the usual analytic operational calculus for bounded operators.

For us, an $\mathscr{2}$-operational calculus is a continuous representation $T(\cdot)$ with compact support of a topological algebra $\mathscr{H}$ of complex-valued functions on the complex plane, in the Banach algebra $B(X)$ of all bounded operators on a given Banach space $X$. It is convenient for our purposes to make some restrictions on $\mathcal{A}$ and on $T(\cdot)$ (see details in $\$ 2$ ). An operator $T$ is "of class $\mathscr{H}$ " if there exists an $\mathscr{H}$-operational calculus $T(\cdot)$ such that $T(z)=T$.

We are concerned with the classification of bounded operators by means of their operational calculus, and with the characterization of certain special classes. We deal mainly with "real operators" (i.e., operators with spectrum on the real line). In this case, we have the following

Classification Theorem. If $T$ is a real operator of class $\mathfrak{A}$ for a homogeneous Banach algebra $\mathfrak{Q}$ (cf. Definition 2.17), then $T$ is of class $C^{n}$ for some $n$.

This allows us to restrict our study to "operators of finite class" (i.e., operators which are of class $C^{n}$ for some $n$ ), as long as $\mathfrak{U}$ has a Banach algebra structure.

Spectral operators of finite type (cf. [4]) are operators of finite class. The simplest necessary and sufficient condition for $T$ to be a real operator of finite class is that $\left\|e^{i t T}\right\|=O\left(|t|^{k}\right)$ for some $k$ ( $t$ real). In fact, the latter condition implies that $T$ is of class $C^{k+2}$. It follows in particular that sums and products of commuting real operators of finite class are of finite class. This shows that these operators are not necessarily spectral (cf. [9] and [14]). However, it is true that operators of class $C$ are spectral if $X$ is weakly complete. This is false in general with $C^{k}(k \geqq 1)$ instead of $C$, even if $X$ is reflexive. In the latter case, we could prove only that "singular" real operators of class $C^{k}$ are spectral of type $k$ (cf. $\S 3$ ).

\footnotetext{
Presented to the Society, January 26, 1964; received by the editors November 23, 1963.

( $\left.{ }^{1}\right)$ This research was partially done while the author was with Princeton University, and was partially supported by a National Science Foundation grant (NSF-GP780) through the Institute for Advanced Study.
} 
In $\S 4$, we derive various characterizations for operators of class $C^{k}$ (for given $k$ ), using either the group $e^{i t T}$ or the resolvent. These results generalize Theorems 4 and 6 in [10].

Theorem. A real operator $T$ is of class $C^{k}$ if and only if there exists a positive number $M$ and a closed finite interval $\Delta$ such that

$$
\left\|\int f(t) e^{i t T} d t\right\| \leqq M\|\hat{f}\|_{k, \Delta}
$$

for all $f \in L_{1, k}$ (the notations are defined in $\$ 4$ ).

For an arbitrary real operator $T$, for $f \in C_{0}^{k}$ (i.e., $f \in C^{k}$ and has compact support), and for $u \geqq 0$, we define a sequence $T_{m}(f ; u)$ of bounded operators $(m=1,2, \cdots)$. We then have the following "constructive" characterization:

Theorem. $T$ is of class $C^{k}$ if and only if, for every $f \in C_{0}^{k}, T_{m}(f ; u)$ converge weakly to a bounded operator $T(f ; u)$, uniformly with respect to $u(u \geqq 0)$, and $\|T(f ; u)\| \leqq M\|f\|_{k, \Delta}(u \geqq 0)$ for some $M>0$ and some finite closed interval $\Delta$.

In this case, the $C^{k}$-operational calculus for $T$ is given by $T(f)=T\left(f_{0} ; 0\right)$, $f \in C^{k}$, where $f_{0}$ is any element of $C_{0}^{k}$ which coincides with $f$ on $\Delta$.

Limits and sums of operators of class $C^{k}$ are studied in \$5. Results of Bade's [1] and Foguel's [6] are generalized.

1. The general operational calculus.

1.1. Definition. A general operational calculus (g.o.c.) is an ordered pair $(\mathscr{A}, T(\cdot))$ which consists of

(i) a topological algebra $\mathfrak{A}$ of complex-valued functions on a subset $\Delta$ of the complex plane, with the ordinary pointwise operations, which contains the restrictions to $\Delta$ of polynomials; and of

(ii) a continuous representation $T(\cdot)$ of $\mathscr{U}$ in $B(X)$, such that $T(1)=I$ ( = the identity operator).

1.2. Definition. An operator $\left({ }^{2}\right) T$ is of class $\mathscr{Q}$ if there exists a g.o.c. $(\mathscr{A}, T(\cdot))$ such that $T(z)=T$; any $T(\cdot)$ with this property will be called an $\mathscr{A}$-operational calculus for $T$. The symbol $T \in(\mathfrak{A})$ stands for the statement " $T$ is of class $\mathfrak{2}$." If $T \in(\mathfrak{H})$, the symbol $T(\cdot)$ will be reserved for any $\mathscr{Q}$-operational calculus for $T$.

Remarks. 1 . The condition $T(1)=I$ is merely a normalization, since, in any case, $T(1)$ is a projection, and $Y=T(1) X$ is an invariant subspace for the representation $T(\cdot)$. The restriction of $T(\cdot)$ to $Y$ defines a g.o.c. satisfying $T(1)=I$ when $X$ is replaced by $Y$.

$\left(^{2}\right)$ The term "operator" is used for "bounded linear operator on $X$ into itself." 
2. Given a g.o.c. $(\mathscr{A}, T(\cdot))$, the operator $T(z)$ is of class $\mathscr{A}$.

1.3. ExAmples. (a) Let $\mathfrak{A}=H(\Delta)$ be the algebra of all complex-valued functions which are locally holomorphic in the open set $\Delta$, with the topology of uniform convergence on every compact subset of $\Delta$.

An operator $T$ is of class $H(\Delta)$ if and only if its spectrum $\sigma(T)$ is contained in $\Delta$. In this case, the representation $T(\cdot)$ is uniquely given by

$$
T(f)=\frac{1}{2 \pi i} \int_{\Gamma} f(z)(z-T)^{-1} d z, \quad f \in H(\Delta),
$$

where $\Gamma$ is an oriented envelope of $\sigma(T)$ with respect to $f$ (cf. [8], Theorem 5.2.5]).

(b) For a compact $\Delta$, let $C(\Delta)$ be the Banach algebra of all continuous complex-valued functions on $\Delta$ with the supremum norm. If $X$ is a Hilbert space, an operator $T$ is of class $C(\Delta)$ if and only if it is similar to a normal operator:

$$
T=Q N Q^{-1}, \quad N N^{*}=N^{*} N .
$$

In this case, the $C(\Delta)$-operational calculus is uniquely given by

$$
T(f)=Q N(f) Q^{-1},
$$

where $f \rightarrow N(f)$ is the usual operational calculus for normal operators.

More generally, if $X$ is a weakly complete Banach space, $(C(\Delta))$ coincides with the family of spectral operators of scalar type with spectrum in $\Delta$ (cf. §3).

(c) Take $\Delta=[0,1], \mathscr{U}=C^{1}(\Delta), X=L^{p}(\Delta) \quad(1 \leqq p \leqq \infty)$ and $T \in B(X)$ defined by:

$$
(T \phi)(x)=x \phi(x)+\int_{x}^{1} \phi(t) d t, \quad \phi \in X, x \in \Delta .
$$

We claim that $T \in(\mathfrak{A})$. Indeed, an $\mathscr{U}$-operational calculus for $T$ is given by:

$$
[T(f) \phi](x)=f(x) \phi(x)+\int_{x}^{1} f^{\prime}(t) \phi(t) d t, \quad f \in \mathcal{A}, \phi \in X, x \in \Delta .
$$

This can be checked by a straightforward calculation.

2. Special operational calculus. In order to get interesting results, we need some restrictions on $\mathfrak{A}$ and $T(\cdot)$ (cf. Definition 1.1).

For a compact subset $K$ of the complex plane, $H(K)$ will denote the algebra of all complex-valued functions which are locally holomorphic in an open neighborhood of $K$, with the usual topology.

Condition 1. If $f \in H(K)$ for a compact $K \neq \emptyset$, then there exists $f_{0} \in \mathscr{A}$ such that $f_{0}=f$ on $\Omega \cap \Delta$, for some neighborhood $\Omega$ of $K$. Roughly speaking, analytic functions belong locally to $\mathfrak{A}$. 
From now on, Condition 1 will replace the assumption that $\mathfrak{A}$ contains the restrictions to $\Delta$ of polynomials, and $\Delta$ will be either the complex plane $\mathbf{C}$ or the real line $\mathbf{R}$, unless otherwise stated. We assume also that the set $\mathfrak{A}_{0}$ of all functions in $\mathfrak{U}$ with compact support is dense in $\mathfrak{U}$.

Fix $f \in \mathscr{A}_{0}$. Suppose $g \in H(\operatorname{Spt} f)$, where Spt $f$ denotes the support of $f$. By Condition 1 , there exists $g_{0} \in \mathfrak{U}$ such that $g_{0}=g$ in a neighborhood of Spt $f$. Define a map

$$
M_{f}: H(\operatorname{Spt} f) \rightarrow \mathfrak{U}
$$

by

$$
M_{f} g=f g_{0}, \quad g \in H(\operatorname{Spt} f) .
$$

$M_{f}$ is well defined. Indeed, if $g_{1} \in \mathfrak{A}$ is such that $g_{1}=g$ in a neighborhood of Spt $f$, then obviously $f g_{0}=f g_{1}$ on Spt $f$, and $f g_{0}=f g_{1}=0$ on [Spt $\left.f\right]^{\prime}$, so that $f g_{0}=f g_{1}$ everywhere (the prime denotes set theoretical complementation).

We can state now

Condition 2. The map $M_{f}: H(\operatorname{Spt} f) \rightarrow \mathfrak{A}$ is continuous (for every $f \in \mathfrak{A}_{0}$ ). The next restrictions are on the representation $T(\cdot)$.

Condition 3. $T(\cdot)$ has compact support (which we denote by $\Sigma$, or $\Sigma_{T}$ when distinction is needed).

$\Sigma$ is the smallest compact $K$ with the property that if $f \in \mathfrak{A}_{0}$ has its support in $K^{\prime}$, then $T(f)=0$.

If $f, g \in \mathscr{U}$ and $f=g$ in a neighborhood of $\Sigma$, then $T(f)=T(g)$; in other words, $T(f)$ depends only on the restriction of $f$ to an arbitrary neighborhood of $\Sigma$.

Let $f \in H(\Sigma)$. By Condition 1 , there exists $f_{0} \in \mathfrak{A}$ such that $f_{0}=f$ in a neighborhood of $\Sigma$. Write $T_{H}(f)=T\left(f_{0}\right)$. The map $T_{H}: H(\Sigma) \rightarrow B(X)$ is well defined; it is obviously a representation; we call it the restriction of $T(\cdot)$ to $H(\Sigma)$. We can (and will) omit the subscript $H$ in $T_{H}$.

Condition 4. The restriction of $T(\cdot)$ to $H(\Sigma)$ is continuous.

2.1. Definition. An operational calculus (o.c.) is a g.o.c. $(\mathfrak{A}, T(\cdot))$ which satisfies Conditions 1-4.

If $\mathscr{Q}$ is a topological algebra satisfying Conditions $1-2$, an operator $T$ is of class $\mathfrak{A}$ if there exists an o.c. $(\mathfrak{A}, T(\cdot))$ such that $T(z)=T$.

An algebra $\mathfrak{A}$ satisfying Conditions $1-2$ is called a basic algebra (cf. [15]).

2.2. Lemma. Let $T$ be an operator of class $\mathfrak{A}$ for a basic $\mathfrak{A}$. Then

$$
\sigma(T)=\Sigma,
$$

where $\Sigma$ denotes the support of any $\mathscr{N}$-operational calculus for $T$.

Remark. Lemma 2.2 does not depend on Condition 4. The following 
proof uses an argument of Foias [7, Proposition 1].

Proof. If $z \notin \Sigma$, there exists $f_{z} \in \mathfrak{U}$ such that $f_{z}(\lambda)=(z-\lambda)^{-1}$ in a neighborhood of $\Sigma$. Hence:

$$
T\left(f_{z}\right)(z I-T)=T\left(f_{z}\right) T(z-\lambda)=T(1)=I,
$$

showing that $z$ is in the resolvent set $\rho(T)$ of $T$. Thus

$$
\sigma(T) \subset \Sigma \text {. }
$$

In order to prove the reversed inclusion, we must show that if $f \in \mathscr{U}_{0}$ has its support in $\rho(T)$, then $T(f)=0$.

For such a function $f$ and for $z \notin \operatorname{Spt} f$, the function $M_{f} g_{z}$ is in $\mathscr{A}$ $\left(g_{z} \in H(\operatorname{Spt} f)\right.$ is defined by $\left.g_{z}(\lambda)=(z-\lambda)^{-1}\right)$. As a function of $z$ from [Spt $f]^{\prime}$ into $\mathcal{A}, M_{f} g_{z}$ is analytic by Condition 2. Therefore $T\left(M_{f} g_{z}\right)$ is an analytic operator-valued function of $z$ on [Spt $f]^{\prime}$.

Let $g_{z, 0} \in \mathscr{U}$ be such that $g_{z, 0}=g_{z}$ in a neighborhood of Spt $f$. We have:

$$
(z I-T) T\left(M_{f} g_{z}\right)=T(z-\lambda) T\left(f g_{z, 0}\right)=T\left(f(z-\lambda) g_{z, 0}\right)=T(f),
$$

because $(z-\lambda) g_{z, 0}=1$ in a neighborhood of Spt $f$, so that $f(z-\lambda) g_{z, 0} \equiv f$. Thus $T\left(M_{f} g_{z}\right)=(z I-T)^{-1} T(f)$ on $\rho(T)$, and therefore $T\left(M_{f} g_{z}\right)$ can be analytically continuated in $\rho(T) \supset \operatorname{Spt} f$. We conclude that $T\left(M_{f} g_{z}\right)$ is entire. Now, for $z \rightarrow \infty$,

$$
T\left(M_{f} g_{z}\right)=(z I-T)^{-1} T(f) \rightarrow 0
$$

in the uniform operator topology. Therefore, by Liouville's Theorem, $T\left(M_{f} g_{z}\right)=0$ for all $z$, and hence $T(f)=0$.

2.3. Lemma. Let $T \in(\mathfrak{A})$ for a basic $\mathfrak{A}$, and let $T(\cdot)$ be an $\mathfrak{A}$-operational calculus for $T$ (cf. Definition 2.1). Then the restriction of $T(\cdot)$ to $H(\Sigma)$ coincides with the analytic operational calculus for $T$.

Proof. By Lemma 2.2, $T_{H}$ is a representation of $H(\sigma(T))$ in $B(X)$. Since $T_{H}(z)=T, T_{H}$ coincides with the analytic operational calculus for $T$ when restricted to rational functions with poles outside $\sigma(T)$. These functions are dense in $H(\sigma(T))$ by Walsh's Theorem [19, p. 16]. The lemma follows now by Condition 4.

2.4. An important example of a basic algebra $\mathfrak{A}$ is the algebra $C^{n}$ of all complex-valued functions on the complex plane with continuous partial derivatives up to the order $n$, and with the topology of uniform convergence on every compact of all partial derivatives of order $\leqq n$. Here $n$ stands for non-negative integers or for $\infty$. Notice that for $C^{n}$, Condition 4 is automatically satisfied by any continuous representation $T(\cdot)$ with compact support.

We need some elementary facts about operators of class $C^{n}$. 
2.5. Lemma. Let $T \in\left(C^{n}\right)$ and let $T(\cdot)$ be a $C^{n}$-operational calculus for $T$. Then $T(f) \in\left(C^{n}\right)$ for every $f \in C^{n}$, and a $C^{n}$-operational calculus for $T(f)$ is given by $[T(f)](g)=T(g \circ f)$, where $g \circ f$ denotes the function $(g \circ f)(x)$ $=g(f(x)), g \in C^{n}$.

Proof. For $f \in C^{n}$ fixed, the map $g \rightarrow T(g \circ f)$ of $C^{n}$ into $B(X)$ is well defined (because $C^{n}$ of $\subset C^{n}$ ). It is clearly a representation of $C^{n}$ in $B(X)$, which takes 1 onto $I$ and $z$ onto $T(f)$. Its continuity follows from the continuity of the map $g \rightarrow g \circ f$ of $C^{n}$ into itself. Finally, its support is contained in $f(\Sigma)$, and is therefore compact ( $\Sigma$ denotes the support of $T(\cdot)$ ).

2.6. For $f \in C^{n}(n<\infty)$ and $\Delta$ compact, $\Delta \subset \mathbf{C}$, we write

$$
\|f\|_{n, \Delta}=\sum_{|j| \leqq n} \sup _{\Delta}\left|D^{j} f\right|,
$$

where $j=\left(j_{1}, j_{2}\right)$ is an ordered pair of non-negative integers, $|j|=j_{1}+j_{2}$, $j !=j_{1} ! j_{2} !$, and

$$
D^{j}=\frac{1}{j !} \frac{\partial^{|j|}}{\partial x^{j_{1}} \partial(i y)^{j_{2}}} .
$$

In case $\Delta \subset \mathbf{R}$, it will be always understood that $j_{2}=0$.

For $\Delta$ compact, $C^{n}(\Delta)$ will denote the algebra of restrictions to $\Delta$ of functions in $C^{n}$, with the topology induced by the norm $\|\cdot\|_{n, \Delta}$.

2.7. Lemma. If $T \in\left(C^{n}\right)$ and $\Delta$ is any compact neighborhood of $\sigma(T)$, then $T \in\left(C^{n}(\Delta)\right)$ in the sense of Definition 1.2. If $n=0, T \in\left(C^{n}(\sigma(T))\right)$. Conversely, if $T \in\left(C^{n}(\Delta)\right)$, then $T \in\left(C^{n}\right)$ and $\sigma(T) \subseteq \Delta$.

Proof. Let $T \in\left(C^{n}\right)$ and let $\Delta$ be a compact neighborhood of $\sigma(T)$. Let $f \in C^{n}(\Delta)$ and let $f_{0} \in C^{n}$ coincide with $f$ on $\Delta$. If $T(\cdot)$ is a $C^{n}$-o.c. for $T$, define $T_{\Delta}(f)=T\left(f_{0}\right)$. By Lemma 2.2, $T_{\Delta}(\cdot)$ is well defined. It is continuous by $[17$, p. 91$]$, and it follows that $T \in\left(C^{n}(\Delta)\right)$.

The case $n=0$ is well known.

Conversely, if $T \in\left(C^{n}(\Delta)\right)$ and $T_{\Delta}(\cdot)$ is a $C^{n}(\Delta)$-o.c. for $T$, a $C^{n}$-o.c. for $T$ is defined by $T(f)=T_{\Delta}(f / \Delta)$, where $f / \Delta$ denotes the restriction of $f$ to $\Delta$. The claim $\sigma(T) \subseteq \Delta$ is checked by noting that $f_{z}(w)=(z-w)^{-1} \in C^{n}(\Delta)$ if $z \notin \Delta$.

For real operators (i.e., operators with spectrum on the real line), the $C^{n}$-o.c. is unique whenever it exists. This follows from the next lemma. For real operators, $C^{n}$ will mean $C^{n}(\mathbf{R})$.

2.8. Lemma. Let $T$ be a real operator of class $C^{n}, n<\infty$. Let $\Delta$ be a compact interval containing $\sigma(T)$ in its interior. Then the $C^{n}(\Delta)$-o.c. for $T$ is unique.

In particular, if $\Delta=[a, b]$ and if $X$ is reflexive, then there exists a unique uniformly bounded operator-valued finitely additive set function $F($.$) on the$ Borel subsets of $[a, b]$, which is countably additive in the strong operator topology, such that 


$$
T_{\Delta}(f)=\sum_{j=0}^{n-1} \frac{f^{(j)}(a)}{j !}(T-a)^{j}+\int_{a}^{b} f^{(n)}(s) d F(s),
$$

$f \in C^{n}(\Delta)$. The integral is understood in the strong operator topology.

Proof. By Lemma 2.7, $T \in\left(C^{n}(\Delta)\right)$. Its $C^{n}(\Delta)$-operational calculus $T_{\Delta}(\cdot)$ is uniquely given on polynomials, which are dense in $C^{n}(\Delta)$ for a compact interval $\Delta$. Since $T_{\Delta}(\cdot)$ is continuous on $C^{n}(\Delta)$, the first part of the lemma is proved.

For $x \in X$ and $x^{*}$ in the adjoint $X^{*}$ of $X$, the map $f \rightarrow x^{*} T_{\Delta}(f) x$ is a continuous linear functional on $C^{n}(\Delta)$. Thus, if $\Delta=[a, b]$, it is uniquely representable in the form:

$$
\dot{x}^{*} T_{\Delta}(f) x=\sum_{j=0}^{n-1} c_{j}\left(x, x^{*}\right) f^{(j)}(a)+\int_{a}^{b} f^{(n)}(s) d \mu\left(s \mid x, x^{*}\right),
$$

where $c_{j}\left(x, x^{*}\right) \in \mathbf{C}$ and $\mu$ is a regular Borel measure on $[a, b]$ (cf. $[5$, p. 344]). Taking $f_{j}(t)=(t-a)^{j} / j$ !, we obtain

$$
c_{j}\left(x, x^{*}\right)=\frac{1}{j !} x^{*}(T-a)^{j} x .
$$

Now, for $f \in C_{0}^{n}(\Delta)=\left\{f \in C^{n}(\Delta) \mid f^{(j)}(a)=0,0 \leqq j \leqq n-1\right\}$, we have

$$
x^{*} T_{\Delta}(f) x=\int_{a}^{b} f^{(n)}(s) d \mu\left(s \mid x, x^{*}\right) .
$$

When $f$ ranges in $C_{0}^{n}(\Delta), g=f^{(n)}$ ranges in the entire space $C(\Delta)$. Therefore

$$
\begin{aligned}
\operatorname{var} \mu & =\sup \left\{\left|\int_{a}^{b} g(s) d \mu\right|, g \in C(\Delta), \max _{\Delta}|g|=1\right\} \\
& =\sup \left\{\left|x^{*} T_{\Delta}(f) x\right|, f \in C_{0}^{n}(\Delta), \max _{\Delta}\left|f^{(n)}\right|=1\right\} .
\end{aligned}
$$

But for $f \in C_{0}^{n}(\Delta)$ such that $\max _{\Delta}\left|f^{(n)}\right|=1$, we have

$$
\left|f^{(n-i)}(t)\right|=\left|\frac{1}{(i-1) !} \int_{a}^{t}(t-s)^{i-1} f^{(n)}(s) d s\right| \leqq \frac{(t-a)^{i}}{i !} \quad(a \leqq t \leqq b),
$$

so that $\|f\|_{n, \Delta} \leqq(1+b-a)^{n} / n$ !. Hence

$$
\left|\mu\left(\delta \mid x, x^{*}\right)\right| \leqq K\|x\|\left\|x^{*}\right\|
$$

for all Borel subsets $\delta$ of $\Delta$, where $K=\left((1+b-a)^{n} / n !\right)\left\|T_{\Delta}(\cdot)\right\|$ and $\left\|T_{\Delta}(\cdot)\right\|$ is the norm of $T_{\Delta}(\cdot)$ as a continuous map of $C^{n}(\Delta)$ into $B(X)$. Using (3) and the uniqueness of the representation (1), we conclude that $\mu\left(\delta \mid x, x^{*}\right)$ is a bounded bilinear form on $X \times X^{*}$. Since $X$ is reflexive, there exists a unique operator $F(\delta)$ such that $x^{*} F(\delta) x=\mu\left(\delta \mid x, x^{*}\right)$ for all $\delta, x$ and $x^{*} . F(\cdot)$ is finitely additive and uniformly bounded by $K$; it is $\sigma$-additive 
in the weak (and hence in the strong) operator topology. Thus $\int_{a}^{b} f^{(n)}(s) d F(s)$ makes sense, and the lemma follows (using (1) and (2)).

2.9. Lemma. Let $T$ be an operator for which there exists an integer $k \geqq 0$ such that

$$
\left\|T^{n}\right\|=O\left(|n|^{k}\right) \quad \text { for integers } n, \quad|n| \rightarrow \infty .
$$

Then $T$ is of class $C^{k+2}$ and its spectrum lies on the unit circle. Conversely, if $T \in\left(C^{k}\right)$ and $\sigma(T)$ lies on the unit circle, then $\left\|T^{n}\right\|=O\left(|n|^{k}\right)$.

Proof. If $f \in C^{k+2}$ and $t \in \mathbf{R}$, the function $\phi(t)=f\left(e^{i t}\right)$ is periodic (period $2 \pi)$ and $k+2$ times continuously differentiable. Therefore $\phi^{(k)}$ has an absolutely convergent Fourier series

$$
\phi^{(k)}(t)=\sum c_{n} e^{i n t},
$$

and

$$
\sum\left|c_{n}\right| \leqq 4\left(\max _{\mathbf{R}}\left|\phi^{(k)}\right|+\max _{\mathbf{R}}\left|\phi^{(k+2)}\right|\right)
$$

If $\phi(t)=\sum d_{n} e^{i n t}$, then $\left|d_{n}\right|=\left|c_{n}\right| /|n|^{k}(n= \pm 1, \pm 2, \cdots)$. Define

$$
T(f)=\sum_{n=-\infty}^{\infty} d_{n} T^{n}, \quad f \in C^{k+2} .
$$

We have:

$$
\left\|d_{n} T^{n}\right\| \leqq \text { Const }|n|^{k} \mid d_{n} ! \leqq \text { Const }\left|c_{n}\right|
$$

Since $\sum\left|c_{n}\right|<\infty$, the series defining $T(f)$ converges "absolutely" in the uniform operator topology. Thus $T(f)$ is well defined. Obviously, the support of $T(\cdot)$ lies on the unit circle; furthermore, $T(1)=I$ and $T(z)=T$. A direct check shows that the map $f \rightarrow T(f)$ is a homomorphism of the algebra $C^{k+2}$ into $B(X)$; its continuity follows from (1) and (3):

$$
\|T(f)\| \leqq \text { Const } \sum\left|c_{n}\right| \leqq \text { Const }\|f\|_{k+2, \Delta},
$$

where $\Delta$ denotes the unit circle.

We conclude that $T \in\left(C^{k+2}\right)$ and $\Sigma \subseteq \Delta$. Since $\Sigma=\sigma(T)$ by Lemma 2.2, the first part of the lemma is proved. The converse follows from Lemma 2.7.

2.10. Corollary. Let $T_{1}$ and $T_{2}$ be commuting operators with spectrum on the unit circle. Suppose $T_{1} \in\left(C^{k}\right)$ and $T_{2} \in\left(C^{l}\right)$. Then $T_{1} T_{2} \in\left(C^{k+l+2}\right)$.

Proof. Applying the "converse" part of Lemma 2.9, we obtain:

$$
\left\|\left(T_{1} T_{2}\right)^{n}\right\| \leqq\left\|T_{1}^{n}\right\|\left\|T_{2}^{n}\right\|=O\left(|n|^{k+l}\right),
$$

and the corollary follows by using the first part of the same lemma. 
2.11. Lemma. Let $T$ be an operator for which there exists an integer $k \geqq 0$ such that

$$
\left\|e^{i t T}\right\|=O\left(|t|^{k}\right), \quad t \in \mathbf{R},|t| \rightarrow \infty .
$$

Then $T$ is of class $C^{k+2}$ and its spectrum lies on the real line. Conversely, if $T$ is a real operator of class $C^{k}$, then (1) holds.

Proof. $\sigma(T)$ is real by (1) and Corollary 1 in $\left[10\right.$, p. 166]. For $f \in C_{0}^{k+2}(\mathbf{R})$, define $T(f)=\int f(t) e^{i t T} d t$, where $f$ is the Fourier transform of $f, f(t)$ $=(1 / 2 \pi) \int e^{-i t s} f(s) d s$. It follows from (1) that the integral defining $T(f)$ converges absolutely and $\|T(f)\| \leqq$ Const $(b-a)\left(\max |f|+\max \left|f^{(k+2)}\right|\right)$, where $(a, b)$ is an interval containing Spt $f$. Hence $f \rightarrow T(f)$ is a continuous linear map of $C_{0}^{k+2}(\mathbf{R})$ into $B(X)$. A straightforward calculation shows that it is multiplicative.

By Equation (2) in [10, p. 164],

$$
T(f)=\lim _{s \rightarrow 0+} \frac{1}{2 \pi i} \int f(t)[R(t-i s ; T)-R(t+i s ; T)] d t .
$$

Suppose $\sigma(T) \subset(-N, N)$ and $f=0$ on $(-N, N)$. Then $T(f)=0$ because $R(t-i s ; T)-R(t+i s ; T) \rightarrow 0$ when $s \rightarrow 0+$ for $t$ not in $(-N, N)$. Now if $f \in C^{k+2}(\mathbf{R})$ and $f_{0} \in C_{0}^{k+2}(\mathbf{R})$ coincides with $f$ on $(-N, N)$, define $T(f)$ $=T\left(f_{0}\right)$. The preceding remark shows that the map $f \rightarrow T(f)$ of $C^{k+2}(\mathbf{R})$ into $B(X)$ is well defined. It is a continuous representation of $C^{k+2}(\mathbf{R})$ on $X$, and it follows readily from (2) that $T(1)=I$ and $T(t)=T$.

The converse follows from Lemma 2.7.

2.12. Corollary. Let $T_{1}$ and $T_{2}$ be commuting real operators of class $C^{k}$ and $C^{l}$ respectively. Then $T_{1}+T_{2}$ is of class $C^{k+l+2}$ and $T_{1} T_{2}$ is of class $C^{m}$ for $m=2 k+2 l+6$.

Proof. Using Lemma 2.11, we obtain:

$$
\left\|e^{i t\left(T_{1}+T_{2}\right)}\right\| \leqq\left\|e^{i t T_{1}}\right\|\left\|e^{i t T_{2}}\right\|=O\left(|t|^{k+l}\right) ;
$$

hence $T_{1}+T_{2} \in\left(C^{k+l+2}\right)$ by the first part of Lemma 2.11.

By Lemma 2.5, if $T \in\left(C^{n}\right)$, also $T^{2}$ and $t T(t \in \mathbf{C})$ are of class $C^{n}$. Writing $T_{1} T_{2}=\frac{1}{2}\left[\left(T_{1}+T_{2}\right)^{2}-T_{1}^{2}-T_{2}^{2}\right]$ and using the result about sums, we conclude that $T_{1} T_{2} \in\left(C^{m}\right)$ for $m=[(k+l+2)+k+2]+l+2=2 k+2 l+6$.

2.13. Definition. An operator is said to be of finite class if it is of class $C^{k}$ for some non-negative integer $k$. In other words, $T \in \bigcup_{k \geqq 0}\left(C^{k}\right)$.

Lemmas 2.9 and 2.11 give simple characterizations of operators of finite class, when the spectrum is either on the unit circle or on the real line. We paraphrase these results.

2.14. THEOREM. Consider the following statements about an operator $T$ : 
1. $T$ is of finite class and $\sigma(T)$ lies on the unit circle.

2. For some integer $k \geqq 0,\left\|T^{n}\right\|=O\left(|n|^{k}\right)$ for integers $n,|n| \rightarrow \infty$.

$1^{\prime}$. $T$ is of finite class and $\sigma(T)$ lies on the real line.

$2^{\prime}$. For some integer $k \geqq 0,\left\|e^{i t T}\right\|=O\left(|t|^{k}\right)$ for $t$ real, $|t| \rightarrow \infty$.

Then $1 \Longleftrightarrow 2$ and $1^{\prime} \Longleftrightarrow 2^{\prime}$.

We paraphrase also Corollaries 2.10 and 2.12:

2.15. Corollary. Sums and products of commuting real operators of finite class are operators of finite class. Products of commuting operators of finite class with spectrum on the unit circle are operators of finite class.

2.16. Remarks. Operators which satisfy Condition 2 in Theorem 2.14 have been studied by several authors. E. R. Lorch [13] and B. Sz.-Nagy [16] deal with the special case $k=0$ in reflexive Banach spaces or in Hilbert space. Lorch calls such operators "weakly almost periodic transformations," while Sz.-Nagy calls them "uniformly bounded transformations." F. Wolf [21] has a result which is equivalent to Lemma 2.9 when $X$ is reflexive. G. K. Leaf [12] studies the class of operators satisfying $\left\|T^{n}\right\|=o(|n|)$.

If $T$ is such that $\left\|e^{i t T}\right\|=O(1)$ for real $t$, Lemma 2.11 asserts that $T$ is of class $C^{2}$. In Hilbert space, this assertion can be improved, namely, $C^{2}$ can be replaced by $C$ (see Theorem 5 in [10]). Such an improvement cannot be achieved in general Banach spaces, and even not in arbitrary reflexive Banach spaces. Indeed, in the latter case, operators of class $C$ are spectral operators of scalar type (cf. \$3). If it were true that $\left\|e^{i t T}\right\|=O(1)$ implies $T \in(C)$, then it would follow that sums (and products) of commuting spectral operators of scalar type (with real spectrum) are spectral; but this is false in general, even in reflexive Banach spaces (see Kakutani [9] and McCarthy [14]).

We see therefore that $C^{k+2}$ cannot be replaced by $C^{k}$ (in general) in the first statement of Lemma 2.11, and similarly in Lemma 2.9. (Such an improvement is impossible already for $k=0$.) We conjecture that this refinement is possible in Hilbert space (the case $k=0$ is verified, as we have remarked above). We are also inclined to think that, at least if $X$ is weakly complete, $C^{k+2}$ may be replaced by $C^{k+1}$.

We come back to the study of operators of class $\mathscr{U}$ in the sense of Definition 2.1, restricting our attention to real operators. In this case, it is convenient to take $\Delta=\mathbf{R}$. Notice that $\Sigma$ is now defined with respect to $R$.

It is natural to restrict the generality of $\mathscr{U}$ in such a way that if $T \in(\mathscr{U})$, also $t T \in(\mathfrak{U})$ for $t \in \mathbf{R}$. An $\mathfrak{U}$-operational calculus for $t T$ which is consistent with the analytic o.c. may be defined by $f \rightarrow(t T)(f), f \in \mathfrak{A}$, where $(t T)(f)$ $=T\left(f_{t}\right)$ and $f_{t}(x)=f(t x) \quad(t, x \in \mathbf{R})$, provided that $f_{t} \in \mathfrak{A}$ whenever $f \in \mathfrak{A}$ and that the map $f \rightarrow f_{t}$ of $\mathfrak{U}$ into itself is continuous. 
2.17. Definition. Let $\mathfrak{A}$ be a basic algebra. We say that $\mathscr{U}$ is homogeneous if $f \in \mathfrak{U}$ implies $f_{t} \in \mathfrak{U}$ (where $f_{t}(x)=f(t x) ; t, x \in \mathbf{R}$ ) and the map $f \rightarrow f_{t}$ of $\mathfrak{A}$ into itself is continuous.

If $\mathfrak{U}$ is a Banach algebra whose norm topology is not weaker than the topology of pointwise convergence, then the condition " $f \in \mathfrak{U} \Longrightarrow f_{t} \in \mathscr{U}$ " implies that the map $f \rightarrow f_{t}$ is automatically continuous. This is an immediate consequence of the Closed Graph Theorem. The same remark is valid if $\mathscr{U}$ is a semi-simple Banach algebra. Indeed, the map $f \rightarrow f_{t}$ is an algebraic representation of $\mathfrak{U}$ into itself. The closure of its image is semi-simple, as a subalgebra of a commutative semi-simple Banach algebra. Therefore the representation is continuous (see L. H. Loomis, An introduction to abstract harmonic analysis, Van Nostrand, New York, 1953, p. 76).

The following result shows that we may restrict our attention to a very concrete case.

2.18. THeOREM. Let $T$ be a real operator of class $\mathcal{A}$, for a homogeneous Banach algebra $\mathfrak{A}$. Then $T$ is of finite class.

Proof. The main argument of the following proof is adapted from Katznelson's proof of Theorem 5.1 in [11]. Denote by $M_{t}$ and $\|T(\cdot)\|$, respectively, the norms of the continuous maps $f \rightarrow f_{t}$ of $\mathscr{A}$ into itself and $f \rightarrow T(f)$ of $\mathscr{A}$ into $B(X)$. For $n=0, \pm 1, \pm 2, \cdots$, we have:

$$
\left\|e^{i n t T}\right\|=\left\|T\left(e^{i n t x}\right)\right\| \leqq\|T(\cdot)\|\left\|e^{i n t x}\right\| \leqq M_{t}\|T(\cdot)\|\left\|e^{i n x}\right\| .
$$

Define

$$
F(t)=\sup _{n} \frac{\left\|e^{i n t T}\right\|}{\left\|e^{i n x}\right\|}, \quad t \in \mathbf{R} .
$$

We have $0 \leqq F(t) \leqq M_{t}\|T(\cdot)\|$. As the limit of a sequence of continuous functions of $t, F(t)$ belongs to the first class of Baire, and therefore, it has points of continuity. If $t_{0}$ is such a point, then for some $\delta>0$, there exists a constant $H=H\left(t_{0}, \delta\right)$ such that $F(t) \leqq H$ for all $t$ in $\left|t-t_{0}\right|<\delta$. For such $t$, we have:

$$
\begin{aligned}
\left\|\exp \left[i n\left(t-t_{0}\right) T\right]\right\| & \leqq\left\|e^{i n t T}\right\|\left\|e^{-i n t_{0} T}\right\| \\
& \leqq F(t) F\left(t_{0}\right)\left\|e^{i n x}\right\|\left\|e^{-i n x}\right\| \leqq H F\left(t_{0}\right) M_{-1}\left\|e^{i n x}\right\|^{2} \\
& =\text { Const }\left\|e^{i n x}\right\|^{2} .
\end{aligned}
$$

Without loss of generality, we may assume $t_{0}=0$ (otherwise, replace $t$ by $t-t_{0}$ in what follows). Thus, for $|t|<\delta$, there exists a constant $Q=Q(\delta)$ such that

$$
\left\|e^{i n t T}\right\| \leqq Q(\delta)\left\|e^{i n x}\right\|^{2}, \quad|t|<\delta .
$$

For $u \in \mathbf{R}$, choose $n$ to be the smallest integer $\geqq 0$ such that 


$$
t 2^{n}=u \text { and }|t|<\delta .
$$

We obtain:

$$
\begin{aligned}
\left\|e^{i u T}\right\| & =\left\|\exp \left(i 2^{n} t T\right)\right\| \leqq Q(\delta)\left\|\exp \left(i 2^{n} x\right)\right\|^{2} \\
& \leqq Q(\delta)\left(M_{2}^{n}\left\|e^{i x}\right\|\right)^{2} .
\end{aligned}
$$

Since $n \leqq(\log |u| / \log 2)+$ const, we have:

$$
M_{2}^{2 n} \leqq \text { const } \exp \left(2 \frac{\log |u|}{\log 2} \log M_{2}\right)=\text { const }|u|^{h} .
$$

Thus $\left\|e^{i u T}\right\|=O\left(|u|^{k}\right)$ for $k=[h]+1$, and the theorem follows from Theorem 2.14. Q.E.D.

Notice that $T \in\left(C^{m}\right)$, where $m=\left[2 \log _{2} M_{2}\right]+2$.

Consider the chain

$$
\text { (C) } \subset\left(C^{1}\right) \subset\left(C^{2}\right) \subset \cdots
$$

Theorem 2.18 states that this chain exhausts all real operators which are of class $\mathfrak{A}$ for some homogeneous Banach algebra $\mathfrak{A}$. We have therefore a kind of general "classification theorem."

Notice that Example 1.3(c) exhibits an operator in $\left(C^{1}\right)-(C)$. Indeed, we have

$$
\left[e^{i t T} \phi\right](x)=e^{i t x} \phi(x)+i t \int_{x}^{1} e^{i t s} \phi(s) d s,
$$

so that $\left\|e^{i t}\right\| \neq O(1)$.

Theorem 2.18 motivates a more detailed study of operators of finite class. This will be done in the following sections.

3. Operators of finite class and spectral operators. We first paraphrase a well-known fact.

3.1. Proposition. Spectral operators of finite type are operators of finite class. More precisely, if $T$ is spectral of type $m$, then $T$ is of class $C^{n}$ for $n \geqq m$.

Proof. Let $T=S+N$ ( $S$ scalar, $N^{m+1}=0$ ) be the canonical decomposition of the spectral operator $T$. Let $f \rightarrow S(f)$ be the $C$-operational calculus for $S$ :

$$
S(f)=\int f(\lambda) d E(\lambda), \quad f \in C,
$$

where $E(\cdot)$ is the resolution of the identity for $T$. Define

$$
T(f)=\sum_{j=0}^{m} \frac{N^{j}}{j !} S\left(L^{j} f\right), \quad f \in C^{n},
$$

where 


$$
L=\frac{1}{2}\left(\frac{\partial}{\partial x}-i \frac{\partial}{\partial y}\right) \text { and } n \geqq m .
$$

The map $f \rightarrow T(f)$ is a continuous linear map of $C^{n}$ into $B(X)$ (if $n \geqq m$ ) with the properties

$$
T(1)=I, \quad T(z)=T \text { and } \operatorname{Spt} T(\cdot)=\sigma(T) .
$$

Let $f, g \in C^{n}$; let $j, h, l$ denote non-negative integers and, finally, let $\alpha, \beta$ denote ordered pairs of such number. Using Leibnitz's rule, we obtain:

$$
\begin{aligned}
T(f g) & =\sum_{j \leqq m} \frac{N^{j}}{2^{j}} \sum_{|\alpha|+|\beta| \leqq j} S\left(D^{\alpha} f\right) S\left(D^{j} g\right) \\
& =\sum_{h \leqq m} \sum_{l \leqq m} \frac{N^{h+l}}{2^{h+l}} \sum_{|\alpha| \leqq h|\beta| \leqq l} \sum_{\mid} S\left(D^{\alpha} f\right) S\left(D^{j} g\right) \\
& =T(f) T(g) . \quad \text { Q.E.D. }
\end{aligned}
$$

Since sums of commuting spectral operators of finite type are not necessarily spectral (cf. [9] and [14]), it follows from Corollary 2.15 and Proposition 3.1 that the converse of Proposition 3.1 is false in general, even for real operators on a reflexive Banach space. However, the converse is true for $m=0$ and $X$ weakly complete.

3.2. TheOREM. In a weakly complete Banach space, an operator is of class $C$ if and only if it is spectral of scalar type.

Proof (for the "only if" part). By Lemma 2.7, $T \in(C(\Delta))$ for $\Delta=\sigma(T)$, in the sense of Definition 1.2. Let $T(\cdot)$ be a $C(\Delta)$-operational calculus for $T$. For $x \in X$ and $x^{*} \in X^{*}$ fixed, $x^{*} T(\cdot) x$ is a continuous linear functional on $C(\Delta)$. The Riesz Representation Theorem states the existence of a regular Borel measure $\mu=\mu\left(\cdot \mid x, x^{*}\right)$ on $\Delta$ such that

$$
x^{*} T(f) x=\int_{\Delta} f(z) \mu\left(d z \mid x, x^{*}\right), \quad f \in C(\Delta),
$$

and

$$
\left\|x^{*} T(\cdot) x\right\|=\left\|\mu\left(\cdot \mid x, x^{*}\right)\right\|
$$

(the norms are understood in the usual way).

Let $\delta$ be a fixed Borel subset of $\Delta$. The uniqueness of the Riesz representation implies that $\mu\left(\delta \mid x, x^{*}\right)$ is a bilinear form on $X \times X^{*}$, which is bounded according to (2):

$$
\left|\mu\left(\delta \mid x, x^{*}\right)\right| \leqq\left\|\mu\left(\cdot \mid x, x^{*}\right)\right\|=\left\|x^{*} T(\cdot) x\right\| \leqq\|T(\cdot)\|\|x\|\left\|x^{*}\right\|,
$$

where $\|T(\cdot)\|$ is the norm of the continuous map $T(\cdot): C(\Delta) \rightarrow B(X)$.

Let $c_{\delta}$ be the characteristic function of $\delta$, and let $f_{n} \in C(\Delta), 0 \leqq f_{n} \leqq 1$, $f_{n} \uparrow c_{\delta}$. It follows from the uniform boundedness of $\mu\left(\cdot \mid x, x^{*}\right)$ and from the 
Lebesgue Dominated Convergence Theorem that $\left\{x^{*} T\left(f_{n}\right) x\right\}$ is a Cauchy sequence for every $x \in X$ and $x^{*} \in X^{*}$. Since $X$ is weakly complete, $\left\{T\left(f_{n}\right)\right\}$ converges in the weak operator topology to a bounded operator, which we denote by $T\left(c_{\delta}\right)$ or $F(\delta)$. A standard check shows that $F(\delta)$ does not depend on the particular sequence $\left\{f_{n}\right\}$, i.e., $F(\delta)$ is well defined. Also $\|F(\delta)\|$ $\leqq\|T(\cdot)\|$. The multiplicativity of $T(\cdot)$ on $C(\Delta)$ implies its multiplicativity on characteristic functions. Hence, for Borel subsets $\delta$ and $\epsilon$ of $\Delta$,

$$
F(\delta) F(\epsilon)=T\left(c_{\delta}\right) T\left(c_{\epsilon}\right)=T\left(c_{\delta} c_{\epsilon}\right)=T\left(c_{\delta \cap \epsilon}\right)=F(\delta \cap \epsilon) .
$$

We also have $x^{*} F(\delta) x=\mu\left(\delta \mid x, x^{*}\right)$. Therefore $F(\cdot)$ is finitely additive on the Borel subsets of $\Delta, F(\emptyset)=0$ and $F(\sigma(T))=T(1)=I$. The $\sigma$-additivity of $x^{*} F(\cdot) x$ implies the strong $\sigma$-additivity of $F(\cdot)$, so that the integral $\int_{\sigma(T)} f(\lambda) d F(\lambda)$ makes sense in the strong operator topology, and is equal to $T(f)$ according to (1). In particular, $T=T(z)=\int_{o(T)} z d F(z)$, and it follows that $T$ is spectral of scalar type by Lemma 6 in [3].

REMARK. Without the weak completeness hypothesis, the same proof shows that if $T$ is of class $C$, then $T^{*}$ is a scalar type spectral operator of class $X$ (cf. Theorem 18 in [3]).

3.3. Corollary. In Hilbert space, an operator is of class $C$ if and only if it is similar to a normal operator (cf. [20]).

3.4. Theorem. In Hilbert space, an operator is of class $C$ with normdecreasing $C(\sigma(T))$-operational calculus if and only if it is normal (cf. Lemma 2.7).

Proof. It is well known that the operational calculus for normal operators is norm-decreasing as a map of $C(\sigma(T))$ into $B(X)$. Therefore, we have only to prove the "only if" part of the theorem.

Let $T \in(C)$ and let $T(\cdot)$ be a norm-decreasing $C(\sigma(T))$-operational calculus for $T$ (cf. Lemma 2.7). Thus

$$
\|T(f)\| \leqq\|f\|, \quad f \in C(\sigma(T)) .
$$

For $t \in \mathbf{R}$, let $f_{t}(z)=\exp (i t \operatorname{Re} z)$.

We consider the group of operators $T\left(f_{t}\right), t \in \mathbf{R}$. By (1), $\left\|T\left(f_{t}\right)\right\| \leqq 1$, and therefore $\left\|T\left(f_{t}\right)\right\|=1$ because $T\left(f_{t}\right)$ is a group. Using Sz.-Nagy's argument in [16], we conclude that $T\left(f_{t}\right)$ is a group of unitary operators. Its infinitesimal generator is therefore of the form $i H$, with $H$ selfadjoint. Since $f \rightarrow T(f)$ is continuous, we obtain:

$$
\begin{aligned}
i H & =\lim _{t \rightarrow 0} t^{-1}\left[T\left(f_{t}\right)-I\right]=\lim _{t \rightarrow 0} T\left(\frac{e^{i t \text { Kez }}-1}{t}\right) \\
& =T(i \operatorname{Re} z)=i T(\operatorname{Re} z) .
\end{aligned}
$$

Hence $T(\operatorname{Re} z)$ is hermitian. 
Considering the group $T\left(g_{t}\right)$ with $g_{t}(z)=\exp (i t \operatorname{Im} z)$, we show in the same way that $T(\operatorname{Im} z)$ is hermitian. Since $T(\operatorname{Re} z)$ commutes with $T(\operatorname{Im} z)$ and $T=T(z)=T(\operatorname{Re} z)+i T(\operatorname{Im} z)$, we conclude that $T$ is normal.

We proceed now to prove a partial converse of Proposition 3.1 for $m \geqq 1$. This will be achieved in a series of lemmas.

3.5. We consider now operators of class $C^{n}$ for $n \geqq 1$. Since $\operatorname{Re} z$ and $\operatorname{Im} z$ are in $C^{n}$, we can write $T(z)=T(\operatorname{Re} z)+i T(\operatorname{Im} z)$. Now $T(\operatorname{Re} z)$ and $T(\operatorname{Im} z)$ are commuting operators of class $C^{n}$ with real spectrum (cf. Lemma 2.5). This shows that any operator of class $C^{n}$ is of the form $A+i B$, where $A$ and $B$ are commuting real operators of class $C^{n}$. We may therefore restrict our study to real operators of finite class. We recall the convention (cf. \$2.16) that, whenever real operators are involved, $C^{n}$ stands for $C^{n}(\mathbf{R})$ and $\Sigma$ is defined with respect to $R$.

If $\phi$ is a continuous linear functional with compact support on $C^{n}$, then it has a representation of the form:

$$
\phi(f)=\sum_{j \leqq n} \int f^{(j)}(t) d \mu_{j}(t) \quad\left\|\mu_{j}\right\| \leqq\|\phi\|, \quad(0 \leqq j \leqq n),
$$

where $\mu_{j}$ are regular finite Borel measures on $\mathbf{R}$ with supports in a neighborhood of Spt $\phi$ (cf. Theorem XXVII in [17, p. 91]). In general, $\phi$ has many representations of this form. Uniqueness can be obtained by restricting in some ways the kinds of measures entering in (1).

3.6. Definition. A continuous linear functional $\phi$ on $C^{n}$ with compact support is singular if it has at least one representation (1) in which all the measures $\mu_{j}, j \geqq 1$, are singular with respect to Lebesgue measure. Such a representation will be called a singular representation of $\phi$.

3.7. Lemma. If $\phi$ is a singular continuous linear functional on $C^{n}$ with compact support, then it has a unique singular representation.

Proof. Using the notations of [17], we have to show that if $\sum_{j=0}^{n} \mu_{j}^{(j)}=0$ and $\mu_{j}$ are singular for $j \geqq 1$, then $\mu_{j}=0$ for $j=0, \cdots, n$. We have $\left(\sum_{j=1}^{n} \mu_{j}^{(j-1)}\right)^{\prime}=-\mu_{0}$, i.e., the distribution $\sum_{j=1}^{n} \mu_{j}^{(j-1)}$ is a primitive of a measure. By Theorem II, p. 54 in [17], this distribution is a function of bounded variation $g_{1}$.

Next, if $n \geqq 2$, we have $\left(\sum_{j=2}^{n} \mu_{j}^{(j-2)}\right)^{\prime}=-\mu_{1}+g_{1}$. Thus, as a primitive of a measure, $\sum_{j=2 \mu_{j}^{(j-2)}}^{n}$ is a function of bounded variation $g_{2}$. Continuing this process, we get finally:

$$
\mu_{n}^{\prime}=-\mu_{n-1}+g_{n-1},
$$

so that $\mu_{n}$ is a function of bounded variation $g_{n}$. But $\mu_{n}$ is singular; hence $g_{n}=0$ a.e. and $\mu_{n}=0$. Going back to (*), we get $\mu_{n-1}=g_{n-1}$; if $n \geqq 2$, $\mu_{n-1}$ is singular, and it follows as before that $\mu_{n-1}=0$. The same argument 
is used to show that $\mu_{j}=0$ for $j \geqq 1$, and hence also $\mu_{0}=0$, since $\mu_{0}$ $+\mu_{1}^{\prime}+\cdots+\mu_{n}^{(n)}=0$.

3.8. Definition. Let $F($.$) be an operator-valued additive set function$ on the Borel subsets of $\mathbf{R}$, which is countably additive in the weak operator topology. Consider the Borel measures $x^{*} F(\cdot) x$ for $x \in X$ and $x^{*} \in X^{*}$. If all these numerical measures are singular with respect to Lebesgue measure, we say that $F(\cdot)$ is singular.

3.9. Definition. Let $T$ be a real operator of class $C^{n}$. For $x \in X$ and $x^{*} \in X^{*}$ fixed, $x^{*} T(\cdot) x$ is a continuous linear functional on $C^{n}$ with compact support. We say that $T$ is singular if $x^{*} T(\cdot) x$ is singular (cf. Definition 3.6) for every $x, x^{*}$.

3.10. Lemma. Let $T$ be a singular real operator of class $C^{n}$ on a reflexive Banach space. Then there exists a unique ordered set of $n+1$ operator-valued additive set functions $\left\{F_{0}(\cdot), \cdots, F_{n}(\cdot)\right\}$, such that:

(1) $F_{j}(\cdot)$ are uniformly bounded and strongly $\sigma$-additive on the Borel sets of $\mathbf{R}(j=0, \cdots, n)$.

(2) $F_{j}(\cdot)$ have compact support contained in an arbitrary neighborhood of $\Sigma$.

(3) For $j \geqq 1, F_{j}(\cdot)$ are singular.

(4) $T(f)=\sum_{j=0}^{n} \int f^{(j)}(t) d F_{j}(t)$ in the strong operator topology.

Proof. We have

$$
x^{*} T(f) x=\sum_{j=0}^{n} \int f^{(j)}(t) d \mu_{j}\left(t \mid x, x^{*}\right), \quad f \in C^{n},
$$

where $\mu_{j}$ are regular Borel measures on $\mathbf{R}$ with compact support (contained in some compact neighborhood $\Delta$ of $\Sigma$ ) such that $\mu_{j}$ are singular for $j \geqq 1$. The bilinearity of $x^{*} T(f) x$ in $x$ and $x^{*}$ and the uniqueness of the singular representation for $x^{*} T(\cdot) x$ (cf. Lemma 3.7) imply that $\mu_{j}\left(\delta \mid x, x^{*}\right)$ is a bilinear form in $x$ and $x^{*}$, for each fixed Borel set $\delta$ and fixed $j$. Furthermore, $\left|\mu_{j}\left(\delta \mid x, x^{*}\right)\right| \leqq\left\|\mu_{j}\left(\cdot \mid x, x^{*}\right)\right\| \leqq\left\|x^{*} T(\cdot) x\right\| \leqq\|T(\cdot)\|\|x\|\left\|x^{*}\right\|\left({ }^{3}\right)$. Since $X$ is reflexive, there exists a unique operator $F_{j}(\delta)$ with bound $\leqq\|T(\cdot)\|$, such that $\mu_{j}\left(\delta \mid x, x^{*}\right)=x^{*} F_{j}(\delta) x(0 \leqq j \leqq n)$. The lemma follows.

3.11. Lemma. Let $\mu_{j}$ and $\nu_{j}(j=0, \cdots, n)$ be Borel measures on $\mathbf{R}$ such that $\mu_{j}$ and $\nu_{j}$ are singular for $j \geqq 1$. Suppose that

$$
\sum_{j=0}^{n}(i t)^{j} \hat{\mu}_{j}(t)=\sum_{j=0}^{n}(i t)^{j} \hat{\nu}_{j}(t), \quad t \in \mathbf{R} .
$$

Then $\mu_{j}=\nu_{j}(j=0, \cdots, n)$. ( $\hat{\mu}$ denotes the Fourier-Stieltjes transform of $\left.\mu.\right)$

Proof. Let $f_{t}(s)=e^{i t s}(s, t \in \mathbf{R})$. The Fourier transform $\hat{\phi}$ of a continuous linear functional $\phi$ on $C^{n}$ with compact support is defined by $\hat{\phi}(t)=\phi\left(f_{t}\right)$.

$\left({ }^{3}\right)\|T(\cdot)\|$ denotes the norm of the $C^{n}(\Delta)$-o.c. for $T$ (cf. Lemma 2.7). 
If $\phi=\sum_{j=0}^{n} \mu_{j}^{(j)}$ is a representation of $\phi$, then $\hat{\phi}(t)=\sum_{j=0}^{n}(i t)^{j} \hat{\mu}_{j}(t)$. The map $\phi \rightarrow \hat{\phi}$ is 1-1. Indeed, if $f \in L_{1, n}(\mathbf{R})$ (i.e., $t^{j} f(t) \in L_{1}(\mathbf{R})$ for $\left.0 \leqq j \leqq n\right)$, then its Fourier transform $f$ is in $C^{n}$ and

$$
\phi(f)=\int_{R} f(t) \hat{\phi}(t) d t .
$$

Therefore, if $\hat{\phi}=0$, also $\phi(f)=0$ for all $f \in \hat{L_{1, n}}$, and since $\hat{L_{1, n}}$ is dense in $C^{n}$, we have $\phi=0$. In particular, if $\phi$ is singular and $\phi=\sum_{j=0}^{n} \mu_{j}^{(j)}$ is its singular representation, then $\hat{\phi}=0$ implies $\mu_{j}=0$ for $j=0, \cdots, n$. This proves the lemma.

3.12. Lemma. Let $T$ be a real singular operator of class $C^{n}$, and let $F_{j}(\cdot)$ be as in Lemma 3.10. Write $E_{j}(\cdot)=j ! F_{j}(\cdot)$ and $E_{j}(t)=\int e^{i t s} d E_{j}(s) \quad(t \in \mathbf{R})$. Then:

$$
\begin{aligned}
& \hat{E_{l}}(t) \hat{E_{r}} \hat{(u)}=E_{l+r}^{\hat{n}}(t+u), \quad l+r \leqq n, \\
& =0, \quad l+r>n
\end{aligned}
$$

$(t, u \in \mathbf{R} ; l, r=0, \cdots, n)$.

Proof. Taking $f_{t}(s)=e^{i t s}$ in Lemma 3.10 (4), we obtain:

$$
e^{i t} T=\sum_{j=0}^{n} \frac{(i t)^{j}}{j !} E_{j}(t) .
$$

Thus, for any pair $\left(t_{1}, t_{2}\right)$ of pure imaginary numbers,

$$
\begin{aligned}
e^{\left(t_{1}+t_{2}\right) T} & =\sum_{j=0}^{n} \sum_{l=0}^{j} \frac{t_{1}^{l} t_{2}^{j-l}}{l !(j-l) !} \int e^{\left(t_{1}+t_{2}\right) s} d E_{j}(s) \\
& =\sum_{l=0}^{n} \frac{t_{1}^{t}}{l !} \sum_{r=0}^{n-l} \frac{t_{2}^{r}}{r !} \int e^{\left(t_{1}+t_{2}\right) s} d E_{l+r}(s) \\
& =e^{t_{1} T} e^{t_{2} T}=\sum_{l=0}^{n} \frac{t_{1}^{l}}{l !} \int e^{t_{1} s} d E_{l}(s) e^{t_{2} T}
\end{aligned}
$$

Thus:

$$
\sum_{l=0}^{n} t_{1}^{l} \int e^{t_{1}^{s} e^{t_{2} s}} d_{s}\left[\frac{1}{l !} \sum_{r=0}^{n-l} \frac{t_{2}^{r}}{r !} E_{l+r}\right]=\sum_{l=0}^{n} t_{1}^{l} \int e^{t_{1} s} d_{s}\left[\frac{1}{l !} E_{l} e^{t_{2} T}\right],
$$

where $d_{s} \mu$ stands for $d_{\mu}(s)$. For $x \in X, x^{*} \in X^{*}$ and $t_{2}$ fixed, the measures

$$
e^{t_{2} s} d_{s}\left[\frac{1}{l !} \sum_{r=0}^{n-l} \frac{t_{2}^{r}}{r !} x^{*} E_{l+r} x\right]
$$

are singular for $l \geqq 1$, because each measure $x^{*} E_{l+r} x$ is singular for $l \geqq 1$, 
$r \geqq 0$ (cf. Lemma $3.10(3)$ ). Similarly, the measures $(1 / l !) x^{*} E_{l}(\cdot) e^{t_{2} 1} x$ are singular for $l \geqq 1$. Therefore, by Lemma 3.11 ,

$$
e^{t_{2} s} d_{s} \sum_{r=0}^{n-l} \frac{t_{2}^{r}}{r !} E_{l+r}=d_{s} E_{l} e^{t_{2} T}, \quad l=0, \cdots, n .
$$

Multiply both sides by $e^{i t s}(t \in \mathbf{R})$ and integrate with respect to $s$ over $\mathbf{R}$ (integrals are understood in the strong operator topology):

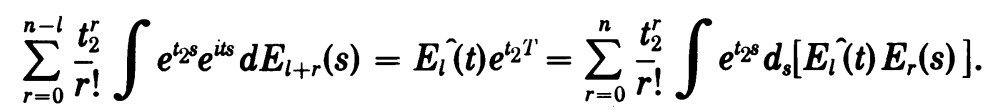

For $x, x^{*}, l$ and $t$ fixed, the measures

$$
x^{*} \hat{E_{l}}(t) E_{r}(\cdot) x=\left\{\left[\hat{E_{l}(t)}\right]^{*} x^{*}\right\} E_{r}(\cdot) x
$$

are singular for $r \geqq 1$ (cf. Lemma 3.10 (3)). Similarly, the measures $e^{i t s} d x^{*} E_{l+r}(s) x$ are singular for $r \geqq 1 \quad(l=0, \cdots, n ; r \leqq n-l)$. Therefore, by Lemma 3.11 (with $t_{2}$ variable), we obtain:

$$
e^{i t s} d E_{l+r}(s)=\hat{E_{l}}(t) d E_{r}(s), \quad r=0, \cdots, n-l,
$$

and

$$
\hat{E_{l}}(t) d E_{r}(s)=0, \quad n-l<r \leqq n,
$$

$(l=0, \cdots, n)$.

Multiplying these equations by $e^{i u s}(u \in \mathbf{R})$ and integrating with respect to $s$ over $\mathbf{R}$, we obtain the identities of the lemma.

3.13. TheOREM. A real operator on a reflexive Banach space is singular of class $C^{n}(n \geqq 1)$ if and only if it is spectral of type $n$ and its nilpotent part $N$ and resolution of the identity $E(\cdot)$ are such that $N E(\cdot)$ is singular.

Proof. If $T$ is a real spectral operator of type $n$, then its $C^{n}$-operational calculus is given by

$$
T(f)=\sum_{j=0}^{n} \int f^{(j)}(s) d\left[\frac{N^{j}}{j !} E(s)\right], \quad f \in C^{n} .
$$

Thus the functional $x^{*} T(\cdot) x$ has a representation $\sum_{j=0}^{n} \mu_{j}^{(j)}$ with $\mu_{j}$ $=\mu_{j}\left(\cdot \mid x, x^{*}\right)=x^{*}\left(N^{j} / j !\right) E(\cdot) x$. For $j \geqq 1$, we write

$$
\mu_{j}(\cdot)=\frac{1}{j !}\left[\left(N^{j-1}\right)^{*} x^{*}\right] N E(\cdot) x .
$$

Therefore $\mu_{j}$ are singular for $j \geqq 1$ if $N E(\cdot)$ is singular. This proves the "if" part of the theorem.

Suppose now that $T$ is a singular real operator of class $C^{n}$. 
Taking $l=r=0$ in Lemma 3.12 , we see that

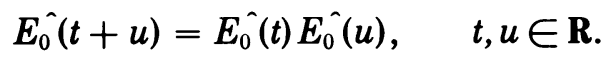

This implies that the map $S(\cdot): f \rightarrow \int f(t) d E_{0}(t)$ is multiplicative over the algebra of functions $f$ of the form $f(s)=\sum_{j=1}^{k} c_{j} \exp \left(i t_{j} s\right) \quad\left(c_{j} \in \mathbf{C}, t_{j} \in \mathbf{R}\right)$. Since this algebra is dense in $C(\sigma(T))$ and since $E_{0}(\cdot)$ is uniformly bounded, $S(\cdot)$ is multiplicative on $C(\sigma(T))$. Taking $f=1$ in Lemma 3.10 (4), we see that $S(1)=E_{0}(\mathbf{R})=T(1)=I$. We conclude that $S(\cdot)$ is a $C$-operational calculus, and the operator $S=S(t)=\int t d E_{0}(t)$ is of class $C$. By Theorem $3.2, S$ is a spectral operator of scalar type (its resolution of the identity is $\left.E_{0}(\cdot)\right)$.

Next, we write $N=E_{1}(\mathbf{R})$.

For $1 \leqq j \leqq n$, take $l=j-1$ and $r=1$ in Lemma 3.12:

$$
E_{j}^{\hat{(}}(t+u)=E_{j-1}^{\hat{n}}(t) E_{1}^{\hat{(}}(u), \quad 1 \leqq j \leqq n .
$$

For $u=0$, this gives $\hat{E_{j}}(t)=E_{j-1}^{\hat{\gamma}}(t) N$. Hence

$$
E_{j}^{\hat{\gamma}}(t)=E_{0}^{\hat{\gamma}}(t) N^{j}, \quad 0 \leqq j \leqq n .
$$

Interchanging the roles of $l$ and $r$, we obtain also

$$
E_{j}^{\hat{\gamma}}(t)=N^{\jmath} E_{0}^{\hat{\gamma}}(t), \quad 0 \leqq j \leqq n .
$$

Equivalently, for $0 \leqq j \leqq n$ and $t \in \mathbf{R}$,

$$
E_{j}(t)=\left[E_{0}(\cdot) N^{j}\right]^{\hat{n}}(t)=\left[N^{j} E_{0}(\cdot)\right]^{\hat{n}}(t) .
$$

Since the Fourier-Stieltjes transform is $1-1$, it follows that

$$
E_{j}(\cdot)=E_{0}(\cdot) N^{\jmath}=N^{\jmath} E_{0}(\cdot), \quad 0 \leqq j \leqq n .
$$

Next, we take $l=n, r=1, t=u=0$ in Lemma 3.12. We obtain $E_{n}(\mathbf{R}) E_{1}(\mathbf{R})$ $=0$; using (1) and remembering that $E_{0}(\mathbf{R})=I$ and $E_{1}(\mathbf{R})=N$, we conclude that $N^{n+1}=0$. We rewrite now equation (4) in Lemma 3.10, using (1) and recalling that $E_{j}(\cdot)=j ! F_{j}(\cdot)$ (write also $E$ for $E_{0}$ ):

$$
T(f)=\sum_{j=0}^{n} \frac{N^{j}}{j !} \int f^{(j)}(s) d E(s), \quad f \in C^{n} .
$$

Taking in particular $f(s)=s$, we get:

$$
T=T(s)=\int s d E(s)+N=S+N .
$$

Since $S$ is spectral of scalar type, $N^{n+1}=0$ and $N$ commutes with $S$ by (1), it follows that $T$ is spectral of type $n$. Furthermore, $N E(\cdot)$ is singular, since $N E(\cdot)=E_{1}(\cdot)$ by (1) (cf. Lemma 3.10 (3)). Q.E.D.

4. Characterizations of real operators of finite class. A simple characteriza- 
tion of real operators of finite class is given in Theorem 2.14 in terms of a growth condition on the group $e^{i t T}, t \in \mathbf{R}$. Our purpose in this section is to characterize $a$ given class $\left(C^{n}\right)$ of real operators. In this case, the necessary condition $\left\|e^{i t T}\right\|=O\left(|t|^{n}\right)$ and the sufficient condition $\left\|e^{i t T}\right\|=O\left(|t|^{n-2}\right)$ (for $n \geqq 2$ ) do not match together (cf. Lemma 2.11 and Remarks 2.16).

We look first for criteria given in terms of the group $e^{i t T}$. One way of getting such criteria is to consider dense subalgebras of $C^{n}$ on which there is a natural definition of $T(f)$.

For $n=0,1, \ldots$, we denote by $L_{1, n}$ the subspace of $L_{1}(\mathbf{R})$ consisting of those $f \in L_{1}(\mathbf{R})$ for which $t^{j} f(t) \in L_{1}(\mathbf{R})$ for $0 \leqq j \leqq n$. Clearly, if $f \in L_{1, n}$, then its Fourier transform $f$ is in $C^{n}$.

We recall that if $\Delta$ is a compact subset of $\mathbf{R}$, and $f \in C^{n}$,

$$
\|f\|_{n, \Delta}=\sum_{j=0}^{n} \frac{1}{j !} \sup _{\Delta}\left|f^{(j)}\right| .
$$

4.1. THEOREM. The following statements are equivalent for a real operator $T$ :

(1) $T$ is of class $C^{n}$.

(2) There exists a positive number $M$ and a closed finite interval $\Delta$ such that

$$
\left\|\int f(t) e^{i t T} d t\right\| \leqq M\|f\|_{n, \Delta} \quad \text { for all } f \in L_{1, n} .
$$

(3) There exists a positive number $M$ and a closed finite interval $\Delta$ such that, for every real vector $\left(t_{1}, \cdots, t_{m}\right)$ and every complex vector $\left(c_{1}, \cdots, c_{m}\right)$, $m=1,2, \cdots$, the following inequality holds:

$$
\left\|\sum_{j=1}^{m} c_{j} \exp \left(i t_{j} T\right)\right\| \leqq M\left\|\sum_{j=1}^{m} c_{j} \exp \left(i t_{j} t\right)\right\|_{n, \Delta}
$$

When (2) or (3) hold, we have $\sigma(T) \subseteq \subseteq \Delta$.

Proof. (1) $\Longrightarrow(2)$. The $C^{n}$-operational calculus for $T$ is an extension of the analytic o.c., so that $e^{i t T}=T\left(\phi_{t}\right)$, where $\phi_{t}(s)=e^{i t s}(s, t \in \mathbf{R})$. Since $\left\|e^{i t T}\right\|=O\left(|t|^{n}\right)$, the integral $\int f(t) e^{i t T} d t$ converges in the uniform operator topology for $f \in L_{1, n}$. The continuity of the map $T(\cdot)$ implies that

$$
\int f(t) e^{i t} d t=\int f(t) T\left(\phi_{t}\right) d t=T\left(\int f(t) \phi_{t} d t\right)=T(f),
$$

and (2) follows.

$(2) \Longrightarrow(1)$. Let $\hat{L_{1, n}}$ denote the subalgebra of $C^{n}$ consisting of the Fourier transforms of functions in $L_{1, n}$. We define a map $T(\cdot): L_{1, n} \rightarrow B(X)$ by

$$
T(\phi)=\int f(t) e^{\imath t T} d t, \quad \text { where } f \in L_{1, n}, f=\phi .
$$


Condition (2) shows that $T(\phi)$ is well defined as a convergent integral in the uniform operator topology. Since $\hat{L_{1, n}}$ is dense in $C^{n}(\Delta)$, we extend the definition of $T(\cdot)$ to $C^{n}(\Delta)$ by continuity; this is possible in view of (2), and we have

$$
\|T(f)\| \leqq M\|f\|_{n, \Delta} \quad \text { for all } f \in C^{n}(\Delta) .
$$

The map $T(\cdot): C^{n}(\Delta) \rightarrow B(X)$ is linear and continuous. In order to prove its multiplicativity, it is enough to check that $T(\phi \Psi)=T(\phi) T(\Psi)$ for $\phi, \Psi \in L_{1, n}$. Let $f, g \in L_{1, n}$ be such that $f=\phi$ and $g=\Psi$. Then $\phi \Psi$ $=(f * g) \hat{,}$, and by Fubini's theorem:

$$
\begin{aligned}
T(\phi \Psi) & =\int(f * g)(t) e^{u t} d t=\iint f(t-s) g(s) d s e^{i t T} d t \\
& =\int f(t) e^{i t T} d t \cdot \int g(s) e^{i s T} d s=T(\phi) T(\Psi) .
\end{aligned}
$$

The use of Fubini's theorem is justified, since (2) implies that $\left\|e^{i t T}\right\|$ $=O\left(|t|^{n}\right)$. Indeed, as we have already remarked, (2) implies the convergence of the integral $\int f(t) e^{i t T} d t$ in the uniform operator topology (for $\left.f \in L_{1, n}\right)$. Therefore, for unit vectors $x$ and $x^{*}$, we have:

$$
\left|\int f(t) x^{*} e^{i t T} x d t\right| \leqq M\|f\|_{n, \Delta} \leqq M\|f\|_{1, n},
$$

where $\|f\|_{1, n}=\sum_{j=0}^{n}\left\|t^{j} f(t)\right\|_{1}$ and $\|\cdot\|_{1}$ is the $L_{1}$-norm. Thus the map $f \rightarrow \int f(t) x^{*} e^{i t} x d t$ is a bounded linear functional on the Banach space $L_{1, n}$ (normed by $\|\cdot\|_{1, n}$ ), with bound $\leqq M$. This implies that $\left\|e^{i t T}\right\|=O\left(|t|^{n}\right.$ ).

In order to show that $T(1)=I$ and $T(s)=T$, it is sufficient to check the identity:

$$
T\left(\phi_{t}\right)=e^{i t T} \quad \text { for } \phi_{t}(s)=e^{i t s} \quad(t, s \in \mathbf{R}) .
$$

The wanted result then follows by expanding both sides of equation $(*)$ in powers of $t$.

Choose a sequence $f_{m, t} \in L_{1, n}$ such that $\left\|f_{m, t}\right\|_{1}=1$ and $f_{m, t} \rightarrow \phi_{t}$ in $C^{n}(\Delta)$. Then $f_{m, t}$ converge to the delta measure $\delta_{t}$ concentrated at $t$, in the weakstar topology of measures. We have:

$$
\begin{aligned}
x^{*} T\left(\phi_{t}\right) x & =\lim _{m \rightarrow \infty} \int f_{m, t}(s) x^{*} e^{i s T} x d s \\
& =\int x^{*} e^{i s T} x d \delta_{t}(s)=x^{*} e^{i t T} x .
\end{aligned}
$$

We conclude that $T$ is of class $C^{n}(\Delta)$; hence $T \in\left(C^{n}\right)$ (cf. Lemma 2.7). The equivalence $(1) \Leftrightarrow(3)$ is proved in a similar way. 
Remarks. We may look at Conditions (2) and (3) as growth conditions on averages of $e^{i t T}$ (compare with Theorem 2.14). For $n=0$ and $X$ reflexive, Theorem 4.1 reduces to Theorem 6 in [10].

Other criteria of the same kind may be obtained by considering other dense subalgebras of $C^{n}(\Delta)$. For example, we have the following criterion: A real operator $T$ is of class $C^{n}$ if and only if there exists a positive number $M$ and a finite closed interval $\Delta$ such that $\|p(T)\| \leqq\|p(t)\|_{n, \Delta}$ for every polynomial $p(t)$.

4.2. We give now a characterization of real operators of class $C^{n}$ which is "constructive" in the sense that it exhibits the $C^{n}$-operational calculus in an explicit form.

Let $C_{0}^{n}$ be the subalgebra of $C^{n}$ consisting of all functions in $C^{n}$ which have compact support.

For $u \geqq 0 ; t, v \in \mathbf{R}$ and $m=1,2, \cdots$, let

$$
K_{m}(t, u, v)=\frac{1}{2 \pi} \exp -\left[(v / m)^{2}+u|v|+i v t\right]
$$

and

$$
G_{m}(t, u)=\int_{\mathbf{R}} K_{m}(t, u, v) e^{i v T} d v
$$

where $T$ is an arbitrary real operator. The integral defining $G_{m}$ converges in the uniform operator topology.

If $f \in C_{0}^{n}$, let

$$
T_{m}(f ; u)=\int_{\mathbf{R}} f(t) G_{m}(t, u) d t \quad(u \geqq 0 ; m=1,2, \cdots) .
$$

We can state now a criterion for $T \in\left(C^{n}\right)$ in terms of the sequence of $\operatorname{maps} T_{m}: C_{0}^{n} \times[0, \infty) \rightarrow B(X)$.

4.3. Theorem. A real operator $T$ is of class $C^{n}$ if and only if, for every $f \in C_{0}^{n}$, the sequence $T_{m}(f ; u)$ converges weakly to an operator $T(f ; u)$ (when $m \rightarrow \infty)$, uniformly with respect to $u(u \geqq 0)$, and, for some positive constant $M$ and some compact interval $\Delta,\|T(f ; u)\| \leqq M\|f\|_{n, \Delta}(u \geqq 0)$.

In this case, the $C^{n}$-operational calculus for $T$ is given by $T(f)=T\left(f_{0} ; 0\right)$, $f \in C^{n}$, where $f_{0} \in C_{0}^{n}$ is such that $f_{0}=f$ on $\Delta$.

Proof. Sufficiency. For $u \geqq 0$, we have the identity $T(f ; u)=T(g ; u)$ whenever $f, g \in C_{0}^{n}$ are such that $f=g$ on $\Delta$. This follows from the inequality $\|T(f ; u)\| \leqq M\|f\|_{n, \Delta}$. We may therefore extend the definition of $T(f ; u)$ to $C^{n}$ by setting

$$
T(f ; u)=T\left(f_{0} ; u\right), \quad f \in C^{n},
$$


where $f_{0} \in C_{0}^{n}$ is such that $f_{0}=f$ on $\Delta$.

Let $f_{t}(s)=e^{i t s}(s, t \in \mathbf{R})$. For $u>0$, we have the identity

$$
T\left(f_{t} ; u\right)=e^{-u|t|} e^{i t T}, \quad t \in \mathbf{R}
$$

(cf. Lemma 5 in [10] and the following remark there).

Now, for $f \in C_{0}^{n}$ fixed, and $m=1,2, \ldots, T_{m}(f ; u)$ is a continuous function of $u$ in the uniform operator topology. Since $x^{*} T_{m}(f ; u) x \rightarrow x^{*} T(f ; u) x$ uniformly with respect to $u(u \geqq 0)$, it follows that $T(f ; u)$ is weakly right continuous at $u=0$. According to the convention made at the beginning of the proof, this is true for $f \in C^{n}$ as well. In particular, taking $f=f_{t}$, we get $T\left(f_{t} ; 0\right)=\lim _{u \rightarrow 0+} T\left(f_{t} ; u\right)=e^{i t T}$ (by $\left.(2)\right)$.

We check now that $T$ satisfies Condition (3) in Theorem 4.1. Since the map $f \rightarrow T(f ; 0)$ is obviously linear, we have (for $c_{j} \in \mathbf{C}$ and $\left.t_{j} \in \mathbf{R} ; j=1, \ldots, N\right)$ :

$$
\left\|\sum c_{j} e^{i t_{j}} T_{\|}=\right\| \sum c_{j} T\left(f_{t j} ; 0\right)\|=\| T\left(\sum c_{j} f_{t j} ; 0\right)\|\leqq M\| \sum c_{j} f_{t_{j}} \|_{n, \Delta} .
$$

Necessity. Let $\Delta$ be a compact interval containing $\Sigma$ in its interior. Fix $x \in X$ and $x^{*} \in X^{*}$. We have:

$$
x^{*} T(f) x=\sum_{j=0}^{n} \int f^{(j)}(s) d \mu_{j}\left(s \mid x, x^{*}\right), \quad f \in C^{n},
$$

where $\mu_{j}$ are regular Borel measures with supports in $\Delta$ and

$$
\left\|\mu_{j}\right\| \leqq\|T(\cdot)\|\|x\|\left\|x^{*}\right\|
$$

(cf. footnote 3).

In particular,

$$
x^{*} e^{i t T} x=\sum_{j=0}^{n}(i t)^{j} \int e^{i t s} d \mu_{j}\left(s \mid x, x^{*}\right),
$$

so that

$$
\begin{aligned}
x^{*} G_{m}(t, u) x & =\sum_{j=0}^{n} \iint K_{m}(t, u, v)(i v)^{j} e^{i s} d v d \mu_{j}\left(s \mid x, x^{*}\right) \\
& =\sum_{j=0}^{n} \int\left(L_{m} * g_{u}\right)^{(j)}(s-t) d \mu_{j}\left(s \mid x, x^{*}\right),
\end{aligned}
$$

where $L_{m}(t)=(2 \pi)^{-1 / 2} m e^{-(m t)^{2}}$ and $g_{u}$ is the measure on $\mathbf{R}$ defined as follows: for $u=0, g_{u}=\delta$ (the delta measure at the origin); for $u>0, g_{u}$ is absolutely continuous with density function $\left(u /\left(u^{2}+t^{2}\right)\right) / \pi$. Notice that var $g_{u}=1$ for $u \geqq 0$.

Now, for $f \in C_{0}^{n}$, we obtain by integrating by parts: 


$$
\begin{aligned}
x^{*} T_{m}(f ; u) x & =\sum_{j=0}^{n} \iint f^{(j)}(t)\left(L_{m} * g_{u}\right)(s-t) d t d \mu_{j}\left(s \mid x, x^{*}\right) \\
& =\sum_{j=0}^{n} \int\left(L_{m} * f^{(j)}(t) d\left(g_{u} * \mu_{j}\right)(t) .\right.
\end{aligned}
$$

Since $f$ has compact support and $f \in C^{n}$, Theorem 1.1.1 in [2] implies that $L_{m} * f^{())} \rightarrow f^{())}$uniformly on $\mathbf{R}$ (for $m \rightarrow \infty ; j=0, \cdots, n$ ). Since var $g_{u}=1$ and $\operatorname{var} \mu_{j} \leqq\|T(\cdot)\|\|x\|\left\|x^{*}\right\|$, we obtain:

$$
\begin{aligned}
\left|x^{*} T_{m}(f ; u) x-\sum_{j=0}^{n} \int f^{(j)}(t) d\left(g_{u} * \mu_{j}\right)(t)\right| \\
\leqq \sum_{j=0}^{n}\left\|L_{m} * f^{(j)}-f^{())}\right\|_{\infty} \cdot \operatorname{var}\left(g_{u} * \mu_{j}\right) \\
\leqq\|T(\cdot)\|\|x\|\left\|x^{*}\right\| \sum_{j=0}^{n}\left\|L_{m} * f^{())}-f^{(j)}\right\|_{\infty} \rightarrow 0
\end{aligned}
$$

when $m \rightarrow \infty$, uniformly in $u(u \geqq 0)$.

But

$$
\begin{aligned}
\sum_{j=0}^{n} \int f^{(j)}(t) d\left(g_{u} * \mu_{j}\right)(t) & =\sum_{j=0}^{n} \int\left(f^{(j)} * g_{u}\right) d \mu_{j} \\
& =\sum_{j=0}^{n} \int\left(f * g_{u}\right)^{(j)} d_{\mu_{j}}=x^{*} T\left(f * g_{u}\right) \dot{x} .
\end{aligned}
$$

Hence $T_{m}(f ; u)$ converges to $T\left(f * g_{u}\right)$ in the weak operator topology, uniformly with respect to $u, u \geqq 0$. Therefore $T(f ; u)=T\left(f * g_{u}\right)$. Since var $g_{u}$ $=1$, we have $\left\|f * g_{u}\right\|_{n, \Delta} \leqq\|f\|_{n, \Delta}$, so that

$$
\|T(f ; u)\|=\left\|T\left(f * g_{u}\right)\right\| \leqq\|T(\cdot)\|\left\|f * g_{u}\right\|_{n, \Delta} \leqq\|T(\cdot)\|\|f\|_{n, \Delta} .
$$

Finally, $T(f ; 0)=T(f * \delta)=T(f)$ for $f \in C_{0}^{n}$, and the proof is complete.

4.4. ExAmple. Let $T$ be as in Example 1.3 (c). We find that

$$
\left(e^{i t T} f\right)(x)=e^{i t x} f(x)+i t \int_{x}^{1} e^{i t s} f(s) d s,
$$

$f \in L^{p}(0,1), 0 \leqq x \leqq 1$. Let $\phi \in C_{0}^{1}$. A straightforward calculation leads to the identity:

$$
\begin{aligned}
{\left[T_{m}(\phi ; 0) f\right](x)=} & \frac{1}{(2 \pi)^{1 / 2}} \int \phi(u+x) m e^{-(m u)^{2}} d u f(x) \\
& +\frac{1}{(2 \pi)^{1 / 2}} \int_{x}^{1} \int \phi^{\prime}(u+s) m e^{-(m u)^{2}} d u f(s) d s
\end{aligned}
$$


Applying Theorem 1.1.1 in [2], we obtain:

$$
\begin{aligned}
{[T(\phi ; 0) f](x) } & =\lim _{m}\left[T_{m}(\phi ; 0) f\right](x) \\
& =\phi(x) f(x)+\int_{x}^{1} \phi^{\prime}(s) f(s) d s .
\end{aligned}
$$

This is precisely the o.c. proposed without justification in $\$ 1.3$.

4.5. Theorem (Characterization in terms of the resolvent, case $n=0)$. A real operator $T$ is of class $C$ if and only if the integral

$$
J\left(s \mid x, x^{*}\right)=\int\left|x^{*}[R(t-i s ; T)-R(t+i s ; T)] x\right| d t
$$

is uniformly bounded when $s \rightarrow 0$, for unit vectors $x$ and $x^{*}$.

Remark. This is a generalization to nonreflexive Banach spaces of the last part of Theorem 6 in [10].

Proof. Necessity. For $s>0$ and $u, t \in \mathbf{R}$, the functions $(t-u \pm i s)^{-1}$, considered as functions of $u$, are in $C$, and $R(t \pm i s ; T)=T_{u}\left((t-u \pm i s)^{-1}\right)$, where the subscript $u$ of $T(\cdot)$ indicates that $(t-u \pm i s)^{-1}$ is considered as a function of $u$. Thus:

$$
\begin{aligned}
J\left(s \mid x, x^{*}\right) & =\int\left|x^{*} T_{u}\left(\frac{2 i s}{(t-u)^{2}+s^{2}}\right) x\right| d t \\
& =2 \int\left|\int \frac{s}{(t-u)^{2}+s^{2}} d \mu\left(u \mid x, x^{*}\right)\right| d t \\
& \leqq 2 \iint \frac{s d t}{(t-u)^{2}+s^{2}} d|\mu|\left(u \mid x, x^{*}\right) \leqq 2 \pi \operatorname{var} \mu\left(\cdot \mid x, x^{*}\right) \\
& \leqq 2 \pi\|T(\cdot)\|\|x\|\left\|x^{*}\right\|,
\end{aligned}
$$

where $\mu\left(\cdot \mid x, x^{*}\right)$ is the measure corresponding to $x^{*} T(\cdot) x$ as a continuous linear functional on $C(\sigma(T))$ and $\|T(\cdot)\|$ is the norm of the $C(\sigma(T))$-o.c. for $T$.

Sufficiency. Let $\Delta$ be a compact interval containing $\sigma(T)$ in its interior. For $s>0$ and $u \in \mathbf{R}$, we have:

$$
\begin{aligned}
e^{-s|u|} e^{i u T} & =\frac{1}{2 \pi i} \int_{\mathbf{R}} e^{i u t}[R(t-i s ; T)-R(t+i s ; T)] d t \\
& =\frac{1}{2 \pi i} \int_{\Delta}+O_{1}(s),
\end{aligned}
$$

where $\left\|O_{1}(s)\right\| \leqq$ Const $s$ (the constant depends on $\Delta$ and $T$, but not on $u$ ) and all the integrals are taken in the uniform operator topology. Applying 
Condition (*), we conclude that $e^{i u T}$ is uniformly bounded for $u \in \mathbf{R}$. Therefore the integral

$$
\int f(u) e^{-s|u|} e^{i u T} d u, \quad f \in L_{1}(\mathbf{R}),
$$

exists in the uniform operator topology, and (by (1) and Fubini's theorem) is equal to

$$
\frac{1}{2 \pi i} \int_{\Delta} f(t)[R(t-i s ; T)-R(t+i s ; T)] d t+O_{2}(s),
$$

where $\left\|O_{2}(s)\right\| \leqq$ Const $\|f\|_{1} s$. Hence, for unit vectors $x$ and $x^{*}$,

$$
\left|x^{*} \int f(u) e^{-s|u|} e^{i u T} d u x\right| \leqq \frac{1}{2 \pi} J\left(s \mid x, x^{*}\right) \cdot \sup _{\Delta}|f|+\left\|O_{2}(s)\right\| .
$$

Since $e^{i u T}$ is uniformly bounded, the left-hand side converges to

$$
\left|x^{*} \int f(u) e^{i u T} d u x\right|
$$

when $s \rightarrow 0+$ (by Lebesgue's Dominated Convergence Theorem). Condition (*) implies therefore that $\left\|\int f(u) e^{i u T} d u\right\| \leqq M \sup _{\Delta}|f|(2 \pi M$ is a uniform bound for $J\left(s \mid x, x^{*}\right)$ when $\left.s \rightarrow 0\right)$; hence $T \in(C)$ by Theorem 4.1.

4.6. REMARKS. If $T$ is a real operator of class $C$, then $T(f)=\int f(t) e^{i t T} d t$ for $f \in L_{1}(R)$ (cf. proof of Theorem 4.1). Thus:

$$
\begin{aligned}
T(f) & =\lim _{s \rightarrow 0+} \int f(t) e^{-s|t|} e^{i t T} d t \\
& =\lim _{s \rightarrow 0+} \frac{1}{2 \pi i} \int f(t)[R(t-i s ; T)-R(t+i s ; T)] d t
\end{aligned}
$$

in the uniform operator topology. The last integral is over any (finite or infinite) interval containing $\sigma(T)$ in its interior. Since $L_{1}(R)^{-}$is dense in $C(\Delta)$ for any compact interval $\Delta$, Condition $(*)$ implies that

$$
T(f)=\lim _{s \rightarrow 0+} \frac{1}{2 \pi i} \int f(t)[R(t-i s ; T)-R(t+i s ; T)] d t
$$

for all $f \in C$ (remember that $\Sigma=\sigma(T)$ ), the limit being now in the weak operator topology. This is an explicit representation of the $C$-operational calculus for $T$ (for real $T \in(C)$ ), which is well known for the special case of hermitian operators in Hilbert space. Compare with Theorem 2 in [18]. The representation (4), as well as Theorem 4.5, are easily generalized to the case $n>0$ (cf. [18]). 
5. Limits and sums of real operators of finite class. Let $T_{a}$ be a net of operators converging strongly to the operator $T$. If each $T_{a}$ is a real operator of finite class, then $\left\|\exp \left(i t T_{a}\right)\right\|=O\left(|t|^{n_{a}}\right)$ for some non-negative integer $n_{a}$. Suppose that there exists an integer $n \geqq 0$ and a constant $M>0$, both not depending upon $a$, such that

$$
\left\|\exp \left(i t T_{a}\right)\right\| \leqq M|t|^{n}, \quad t \in \mathbf{R} .
$$

Then, by the Uniform Boundedness Theorem, we have also $\left\|e^{i t T}\right\| \leqq M|t|^{n}$, and therefore $T$ is of finite class (in fact, it is of class $C^{n+2}$; cf. Lemma 2.11). In particular, if $T_{a}$ are all of the same class $C^{n}$, the condition

$$
\sup _{a}|t|^{-n}\left\|\exp \left(i t T_{a}\right)\right\|<\infty
$$

is sufficient for $T \in\left(C^{n+2}\right)$. This is quite unsatisfactory, because we cannot conclude by this argument that $T$ is in the same class $C^{n}$ as all the $T_{a}$. A more precise result may be obtained by applying Theorem 4.1.

5.1. Definition. Let $T \in B(X)$ and let $\Delta$ be a compact interval. The $n$th variation of $T$ over $\Delta$ is defined by

$$
v_{n}(T ; \Delta)=\sup \left\|\int_{\mathbf{R}} f(t) e^{i t T} d t\right\|,
$$

where the sup is taken over all $f \in L_{1, n}$ for which $\|f\|_{n, \Delta}=1\left({ }^{4}\right)$.

In general, $v_{n}(T ; \Delta)=\infty$. Theorem 4.1 states that $v_{n}(T ; \Delta)<\infty$ if and only if $T$ is a real operator of class $C^{n}$ (in this case, $\sigma(T) \subseteq \Delta$ ).

Furthermore, since $T(\phi)=\int f(t) e^{i t T} d t$ for $\phi=f$ and $f \in L_{1, n}$, and since $L_{1, n}$ is dense in $C^{n}(\Delta)$, we have:

$$
\begin{aligned}
v_{n}(T ; \Delta) & =\sup \left(\|T(\phi)\| ; \phi \in \hat{L_{1, n}},\|\phi\|_{n, \Delta}=1\right) \\
& =\sup \left(\|T(\phi)\| ; \phi \in C^{n}(\Delta),\|\phi\|_{n, \Delta}=1\right) \\
& =\|T(\cdot)\|,
\end{aligned}
$$

where $T(\cdot)$ is the $C^{n}(\Delta)$-operational calculus for $T \in C^{n}$ (cf. Lemma 2.7) and $\|T(\cdot)\|$ is the norm of $T(\cdot)$ as a continuous linear map of $C^{n}(\Delta)$ into $B(X)$.

5.2. Theorem. Let $T_{a}$ be a net of operators converging strongly to the operator $T$. Suppose that, for some fixed integer $n \geqq 0$ and compact interval $\Delta$, the nth variations of $T_{a}$ over $\Delta$ are uniformly bounded. Then $T$ (as well as all $T_{a}$ ) is of class $C^{n}$ with spectrum in $\Delta$, and $T(f)=\lim T_{a}(f)$ in the strong operator topology for all $f \in C^{n}$.

$\left({ }^{4}\right)$ We set $v_{n}(T ; \Delta)=\infty$ if the integral does not converge in the uniform operator topology for some $f \in L_{1, n}$ with $\|f\|_{n, \Delta}=1$. 
Proof. By Theorem 4.1, $T_{a} \in\left(C^{n}\right)$ and $\sigma\left(T_{a}\right) \subseteq \Delta$. It follows in particular that

$$
\begin{aligned}
\left\|\exp \left(i t T_{a}\right)\right\| & =\left\|T_{a}\left(e^{i t s}\right)\right\| \leqq\left\|T_{a}(\cdot)\right\|\left\|e^{i t s}\right\|_{n, \Delta} \\
& \leqq v_{n}\left(T_{a} ; \Delta\right) \sum_{j=0}^{n}|t|^{j} .
\end{aligned}
$$

There exists therefore a constant $K>0$ which does not depend on $a$ such that $\left\|\exp \left(i t T_{a}\right)\right\| \leqq K\left(|t|^{n}+1\right)$. If $f \in L_{1}(\mathbf{R})$ vanishes outside some finite interval $(-A, A)$ (so that, in particular, $\left.f \in L_{1, n}\right)$, then, by Lebesgue Dominated Convergence Theorem for nets (cf. [5], p. 124),

$$
\left\|\int f(t) e^{i t T} d t x\right\|=\lim _{a}\left\|\int f(t) e^{i t T_{a}} d t x\right\| \leqq M\|x\|\|f\|_{n, \Delta},
$$

where $M=\sup _{a} v_{n}\left(T_{a} ; \Delta\right)<\infty$. Now if $f \in L_{1, n}$, there is a sequence $f_{k}$ $\in L_{1}(\mathbf{R})$ such that (i) each $f_{k}$ vanishes outside some finite interval, and (ii) $\mathcal{S}|t|^{j}\left|f(t)-f_{k}(t)\right| d t \rightarrow 0$ when $k \rightarrow \infty \quad(0 \leqq j \leqq n)$. By (ii), $f_{k} \rightarrow f$ in $C^{n}(\Delta)$. Since $\left\|e^{i t T}\right\| \leqq K\left(|t|^{n}+1\right)$, we have:

$$
\left\|\int f(t) e^{i t T} d t-\int f_{k}(t) e^{i t T} d t\right\| \leqq K \int\left(|t|^{n}+1\right)\left|f(t)-f_{k}(t)\right| d t \rightarrow 0
$$

$(k \rightarrow \infty)$. Therefore:

$$
\begin{aligned}
\left\|\int f(t) e^{i t T} d t\right\| & =\lim _{k}\left\|\int f_{k}(t) e^{i t T} d t\right\| \leqq M \limsup _{k}\left\|f_{k}\right\|_{n, \Delta} \\
& =M\|f\|_{n, \Delta} \text { for all } f \in L_{1, n} .
\end{aligned}
$$

By Theorem 4.1, $T \in\left(C^{n}\right)$. The identity $T(\phi)=\lim T_{a}(\phi)$ is valid (in the strong operator topology) for $\phi \in L_{1, n}$, and therefore for every $\phi \in C^{n}$, since $L_{1, n}$ is dense in $C^{n}(\Delta)$ and $\Sigma=\sigma(T) \subseteq \Delta$ (cf. [5, p. 55, Theorem 18]).

Theorem 5.2 generalizes Theorem 7 in [10] and Theorems 2.3 and 2.6 in [1].

5.3. Sums of operators of finite class. According to Corollary 2.15, sums and products of commuting real operators of finite class are of finite class. In general, this statement is no longer true if we replace "finite class" by "class $C^{n}$ " for some fixed $n$, not even for $n=0$ and $X$ reflexive (cf. [9] and [14]). Consider the special case of a sum $T+S$, where $T$ commutes with $S$, $T$ is real of class $C^{n}$ and $S$ is real of class $C$. By Corollary 2.12, T+S is of class $C^{n+2}$. In order to conclude that $T+S$ is of class $C^{n}$, we need a certain boundedness condition, which is automatically satisfied in Hilbert space. We state first an elementary result.

5.4. Lemma. Let $E_{j}(j=1, \cdots, N)$ be projections such that $E_{j} E_{k}=0$ for 
for $j \neq k$ and $\Sigma E_{j}=I$. Let $T$ be a real operator of class $C^{n}$ which commutes with $E_{j}(j=1, \cdots, N)$. Finally, let $L=T+\sum_{1 \leqq j \leqq N} a_{j} E_{j}, a_{j} \in \mathbf{R}$. Then $L$ is of class $C^{n}$, and its $C^{n}$-operational calculus is given by

$$
L(f)=\sum_{j=1}^{N} T\left(f_{a_{j}}\right) E_{j}, \quad f \in C^{n},
$$

where $f_{a}(t)=f(t+a), t, a \in \mathbf{R}$.

Proof. Define $L(f)$ by (1). The map $f \rightarrow L(f)$ is a continuous linear map of $C^{n}$ into $B(X)$. We have $L(1)=\sum T(1) E_{j}=\sum E_{j}=I$ and $L(t)$ $=\sum T\left(t+a_{j}\right) E_{j}=\sum\left(T+a_{j} I\right) E_{j}=L$. If $f, g \in C^{n}$, then

$$
\begin{aligned}
L(f g) & =\sum T\left([f g]_{a_{j}}\right) E_{j}=\sum T\left(f_{a_{j}}\right) T\left(g_{a_{j}}\right) E_{j} \\
& =\sum_{j} \sum_{k} T\left(f_{a_{j}}\right) T\left(g_{a_{k}}\right) E_{j} E_{k}=L(f) L(g) .
\end{aligned}
$$

We have used here the commutativity of $T$ with $E_{j}$, which, in turn, implies the commutativity of $T(f)$ with $E_{j}$ for every $f \in C^{n}$ and $j=1, \cdots, N$ (this is obvious if $f$ is a polynomial; since polynomials are dense in $C^{n}(\Delta)$ for a compact interval $\Delta$, the result follows). Finally, $L(\cdot)$ has the compact support $\bigcup_{j=1}^{N}\left(\Sigma+a_{j}\right)$.

5.5. TheOREM. Let $T$ and $S$ be commuting real operators. Suppose

(i) $T \in C^{n}$.

(ii) $S$ is spectral of scalar type.

(iii) $\sup \left\|\sum_{1 \leqq j \leqq N} T\left(f_{a_{j}}\right) E\left(\sigma_{j}\right)\right\|<\infty$,

where $E(\cdot)$ is the resolution of the identity for $S$, and the sup is taken over all finite partitions $\left\{\sigma_{1}, \cdots, \sigma_{N}\right\}$ of $\sigma(S)$ into disjoint Borel sets, with arbitrary choice of $a_{j} \in \sigma_{j}$, and over all $f \in C^{n}$ with $\|f\|_{n, \Delta}=1$ ( $\Delta$ is some fixed compact interval).

Then $T+S$ is of class $C^{n}$, and its $C^{n}$-operational calculus is given by

$$
(T+S)(f)=\int T\left(f_{t}\right) d E(t), \quad f \in C^{n}
$$

(the integral exists in the strong operator topology).

Proof. Denote $\pi=\left\{\sigma_{j}, a_{j} \mid j=1, \cdots, N\right\}$, where $\sigma_{j}$ are disjoint Borel subsets of $\sigma(S), \sigma(S)=\bigcup_{\sigma_{j}}$ and $a_{j} \in \sigma_{j}$. Let

$$
L_{\star}=T+\sum_{j=1}^{N} a_{j} E\left(\sigma_{j}\right) .
$$

We have $\lim L_{\pi}=T+S$ in the usual sense. By Lemma 5.4, each $L_{\pi}$ is of class $C^{n}$ and its $C^{n}$-operational calculus is given by 


$$
L_{\pi}(f)=\sum_{j=1}^{N} T\left(f_{a_{j}}\right) E\left(\sigma_{j}\right) .
$$

Condition (iii) means that the $n$th variations of $L_{\pi}$ over $\Delta$ are uniformly bounded. Therefore, by Theorem $5.2, T+S$ is of class $C^{n}$; furthermore $\lim L_{\pi}(f)$ exists in the strong operator topology, and gives us the $C^{n}$-operational calculus for $T+S$. On the other hand, $\lim L_{x}(f)$ is by definition the "strong" integral $\int T\left(f_{t}\right) d E(t)$.

5.6. Corollary. Let $T$ and $S$ be commuting real operators in Hilbert space, which are respectively of class $C^{n}$ and of class $C$. Then $T+S$ is of class $C^{n}$ and equation (*) in Theorem 5.5 gives its o.c.

Proof. By Lemma 4 in [3], there exists a constant $M$ depending only on $S$ such that

$$
\left\|\sum_{j=1}^{N} T\left(f_{a_{j}}\right) E\left(\sigma_{j}\right)\right\| \leqq M \sup _{1 \leqq j \leqq N}\left\|T\left(f_{a_{j}}\right)\right\|
$$

for any collection $\sigma_{j}(1 \leqq j \leqq N)$ of disjoint Borel sets. Let $\Delta$ be a compact interval containing $\Delta_{0}+\sigma(S)$, where $\Delta_{0}$ is a compact interval containing $\sigma(T)$ in its interior. Let $K$ be the norm of the $C^{n}\left(\Delta_{0}\right)$-o.c. for $T$. Taking $\sigma_{j} \subset \sigma(S)$ and $a_{j} \in \sigma_{j}$, we have:

$$
\left\|T\left(f_{a_{j}}\right)\right\| \leqq K\left\|f_{a_{j}}\right\|_{n, \Delta_{0}}=K\|f\|_{n, \Delta_{0}+a_{j}} \leqq K\|f\|_{n, \Delta} .
$$

We conclude that condition (iii) in Theorem 5.5 is satisfied, and the corollary follows.

Theorem 5.5 generalizes Theorem 7 in [6] about sums of commuting spectral operators (for the case of real spectrum). The uniform boundedness of the Boolean algebra of projections generated by the resolutions of the identity $E_{1}$ and $E_{2}$ of $T$ and $S$ respectively ( $T$ and $S$ scalar) implies condition (iii) in Theorem 5.5.

\section{BiBLIOGRAPHY}

1. W. G. Bade, Weak and strong limits of spectral operators, Pacific J. Math. 4 (1954), 393-413.

2. S. Bochner, Harmonic analysis and the theory of probability, Univ. California Press, Berkeley, Calif., 1955.

3. N. Dunford, Spectral operators, Pacific J. Math. 4 (1954), 321-354.

4. _ , A survey of the theory of spectral operators, Bull. Amer. Math. Soc. 64 (1958), 217-274.

5. N. Dunford and J. Schwartz, Linear operators. I, Interscience, New York, 1958.

6. S. R. Foguel, Sums and products of commuting spectral operators, Ark. Mat. 3 (1957), 449-461.

7. C. Foiaş, Une application des distributions vectorielles à la théorie spectrale, Bull. Sci. Math. (2) 84 (1960), 147-158.

8. E. Hille and R. S. Phillips, Functional analysis and semi-groups, Amer. Math. Soc. Colloq. Publ. Vol. 31, Amer. Math. Soc., Providence, R. I., 1957. 
9. S. Kakutani, An example concerning uniform boundedness of spectral measures, Pacific J. Math. 4 (1954), 363-372.

10. S. Kantorovitz, On the characterization of spectral operators, Trans. Amer. Math. Soc. 111 (1964), 152-181.

11. Y. Katznelson, Sur le calcul symbolique dans quelques algebres de Banach, Ann. Sci. Ecole Norm. Sup. (3) 76 (1959), 83-123.

12. G. K. Leaf, A spectral theory for a class of linear operators, Pacific J. Math. 13 (1963), 141-155.

13. E. R. Lorch, The integral representation of weakly almost periodic transformations in reflexive vector spaces, Trans. Amer. Math. Soc. 49 (1941), 18-40.

14. C. A. McCarthy, Commuting Boolean algebras of projections, Pacific J. Math. 11 (1961), 295-307.

15. F. Maeda, Generalized spectral operators on locally convex spaces, Pacific J. Math. 13 (1963), 177-192.

16. B. Sz.-Nagy, On uniformly bounded linear transformations in Hilbert space, Acta Sci. Math. (Szeged) 11 (1947), 152-157.

17. L. Schwartz, Théorie des distributions. I, Actualités Sci. Indust. No. 1091, Hermann, Paris, 1950.

18. H. G. Tillmann, Vector-valued distributions and the spectral theorem for selfadjoint operators in Hilbert space, Bull. Amer. Math. Soc. 69 (1963), 67-71.

19. J. L. Walsh, Interpolation and approximation by rational functions in the complex domain, Amer. Math. Soc. Colloq. Publ. Vol. 20, Amer. Math. Soc., Providence, R. I., 1935.

20. J. Wermer, Commuting spectral measures on Hilbert space, Pacific J. Math. 4 (1954), 355-361.

21. F. Wolf, Operators in Banach space which admit a generalized spectral decomposition, Nederl. Akad. Wetensch. Proc. Ser. A 60 (1957), 302-311.

Princeton University,

Princeton, New Jersey

INSTITUTE FOR ADVANCED STUdY,

Princeton, New Jersey 